\title{
ON SEMICLASSICAL GROUND STATES \\ FOR HAMILTONIAN ELLIPTIC SYSTEM WITH CRITICAL GROWTH
}

\author{
Jian Zhang - Xianhua Tang — Wen Zhang
}

\begin{abstract}
In this paper, we study the following Hamiltonian elliptic system with gradient term and critical growth:

$$
\begin{cases}-\varepsilon^{2} \Delta \psi+\varepsilon b \cdot \nabla \psi+\psi=K(x) f(|\eta|) \varphi+W(x)|\eta|^{2^{*}-2} \varphi & \text { in } \mathbb{R}^{N} \\ -\varepsilon^{2} \Delta \varphi-\varepsilon b \cdot \nabla \varphi+\varphi=K(x) f(|\eta|) \psi+W(x)|\eta|^{2^{*}-2} \psi & \text { in } \mathbb{R}^{N}\end{cases}
$$

where $\eta=(\psi, \varphi): \mathbb{R}^{N} \rightarrow \mathbb{R}^{2}, K, W \in C\left(\mathbb{R}^{N}, \mathbb{R}\right), \varepsilon$ is a small positive parameter and $b$ is a constant vector. We require that the nonlinear potentials $K$ and $W$ have at least one global maximum. Combining this with other suitable assumptions on $f$, we prove the existence, exponential decay and concentration phenomena of semiclassical ground state solutions for all sufficiently small $\varepsilon>0$.
\end{abstract}

\section{Introduction and main results}

We study the following Hamiltonian elliptic system with gradient term and critical growth:

$$
\begin{cases}-\varepsilon^{2} \Delta \psi+\varepsilon b \cdot \nabla \psi+\psi=K(x) f(|\eta|) \varphi+W(x)|\eta|^{2^{*}-2} \varphi & \text { in } \mathbb{R}^{N} \\ -\varepsilon^{2} \Delta \varphi-\varepsilon b \cdot \nabla \varphi+\varphi=K(x) f(|\eta|) \psi+W(x)|\eta|^{2^{*}-2} \psi & \text { in } \mathbb{R}^{N}\end{cases}
$$

2010 Mathematics Subject Classification. 35J50, 58E05.

Key words and phrases. Hamiltonian elliptic systems; semiclassical ground states; concentration; critical growth.

This work is partially supported by the NNSF (Nos. 11601145, 11561370, 11471137) and Hunan University of Commerce Innovation Driven Project for Young Teacher (No. 16QD008). 
where $\eta=(\psi, \varphi): \mathbb{R}^{N} \rightarrow \mathbb{R}^{2}, N \geq 3, \varepsilon>0$ is a small parameter, $2^{*}=$ $2 N /(N-2)$ is the usual critical exponent and $b$ is a constant vector, and $f$ is a superlinear and subcritical nonlinearity. In this paper, we are concerned with the existence, exponential decay and concentration phenomenon of semiclassical ground state solutions of system $\left(\mathcal{P}_{\varepsilon}\right)$.

Systems $\left(\mathcal{P}_{\varepsilon}\right)$ or similar to $\left(\mathcal{P}_{\varepsilon}\right)$ were studied by a number of authors. But most of them focused on the case $b=0$. For example, see [3]-[5], [9], [12], [20], [24], [26], [29], [32], [33], [35]-[38], [40], [41], [43] and the references therein. When $b \neq 0$ and $\varepsilon=1$, there are not so many works on elliptic systems with the gradient term. Zhao and Ding [39] considered the following system:

$$
\begin{cases}-\Delta \psi+b(x) \cdot \nabla \psi+V(x) \psi=H_{\varphi}(x, \psi, \varphi) & \text { in } \mathbb{R}^{N} \\ -\Delta \varphi-b(x) \cdot \nabla \varphi+V(x) \varphi=H_{\psi}(x, \psi, \varphi) & \text { in } \mathbb{R}^{N}\end{cases}
$$

where $b=\left(b_{1}, \ldots, b_{N}\right) \in C^{1}\left(\mathbb{R}^{N}, \mathbb{R}^{N}\right), V \in C\left(\mathbb{R}^{N}, \mathbb{R}\right)$ and $H \in C^{1}\left(\mathbb{R}^{N} \times \mathbb{R}^{2}, \mathbb{R}\right)$. In this case, the appearance of the gradient term in this system brings some difficulties, and the variational framework for the case $b=0$ cannot work any longer. Hence the authors first established suitable variational framework through the studying of the spectrum of operator, and obtained the multiplicity of solution for the non-periodic asymptotically quadratic case by applying the theorems of Bartsch and Ding [6]. Moreover, without the assumption that $H(x, \eta)$ is even in $\eta$, infinitely many geometrically distinct solutions for the periodic asymptotically quadratic case were obtained by using a reduction method. For the periodic superquadratic case, Zhang et al. [42] proved the existence of ground state solution for system (1.1). Recently, Yang et al. [34] considered the non-periodic superquadratic system

$$
\begin{cases}-\Delta \psi+b \cdot \nabla \psi+\psi=H_{\varphi}(x, \psi, \varphi) & \text { in } \mathbb{R}^{N} \\ -\Delta \varphi-b \cdot \nabla \varphi+\varphi=H_{\psi}(x, \psi, \varphi) & \text { in } \mathbb{R}^{N}\end{cases}
$$

with a constant vector $b$. Since the problem is set in unbounded domain with nonperiodic nonlinearities, the $(C)_{c}$-condition does not hold in general. To overcome the difficulty, they first considered certain limit problem related to system (1.2) which is autonomous, and constructed linking levels of the variational functional and proved the $(C)_{c}$-condition.

For small $\varepsilon>0$ the solutions (standing waves) of $\left(\mathcal{P}_{\varepsilon}\right)$ are referred to as semiclassical states, which describe the transition from quantum mechanics to classical mechanics when the parameter $\varepsilon$ goes to zero, and possess an important physical interest. For such case, the asymptotic behavior of semiclassical states, such as concentration, exponential decay, etc., is a very interesting problem in mathematics and physics. To the best of our knowledge, there is only a few works concerning the existence and concentration phenomena of semiclassical states. 
Very recently, Zhang et al. [44] studied the following singularly perturbed system:

$$
\begin{cases}-\varepsilon^{2} \Delta \psi+\varepsilon b \cdot \nabla \psi+\psi=K(x)|\eta|^{p-2} \varphi & \text { in } \mathbb{R}^{N}, \\ -\varepsilon^{2} \Delta \varphi-\varepsilon b \cdot \nabla \varphi+\varphi=K(x)|\eta|^{p-2} \psi & \text { in } \mathbb{R}^{N},\end{cases}
$$

with $p \in\left(2,2^{*}\right)$. The authors proved the existence of semiclassical ground state solutions of system (1.3), and also shown that these solutions converge to the ground state solutions of the associated limit problem and concentrate to the maxima point of nonlinear potential $K$ as $\varepsilon \rightarrow 0$. For the general subcritical case with competing potentials, see [45]. However, there are no results studying the existence and concentration phenomena of semiclassical solutions for the critical growth case. So, it is quite natural to ask if certain similar properties about semiclassical solutions can be obtained for the critical case. As we will see, the answer is affirmative. In the present paper we will investigate system $\left(\mathcal{P}_{\varepsilon}\right)$ with critical growth and more general subcritical nonlinearity $f$. More precisely, we prove the existence, concentration, exponential decay and $H^{2}$-convergence of the semiclassical ground state solutions to system $\left(\mathcal{P}_{\varepsilon}\right)$. Compared with the study of system (1.3), the study of system $\left(\mathcal{P}_{\varepsilon}\right)$ becomes more complicated due to the effect of the critical growth and the general subcritical nonlinearity, so the present argument seems to be more delicate.

Let us now describe the results of the present paper. For notational convenience, let

$$
\mathcal{J}=\left(\begin{array}{cc}
0 & -1 \\
1 & 0
\end{array}\right), \quad \mathcal{J}_{0}=\left(\begin{array}{ll}
0 & 1 \\
1 & 0
\end{array}\right)
$$

and $\mathcal{S}_{\varepsilon}=-\varepsilon^{2} \Delta+1$. We denote

$$
A_{\varepsilon}:=\mathcal{S}_{\varepsilon} \mathcal{J}_{0}+\varepsilon b \cdot \nabla \mathcal{J}=\left(\begin{array}{cc}
0 & -\varepsilon^{2} \Delta-\varepsilon b \cdot \nabla+1 \\
-\varepsilon^{2} \Delta+\varepsilon b \cdot \nabla+1 & 0
\end{array}\right) .
$$

Then system $\left(\mathcal{P}_{\varepsilon}\right)$ can be rewritten as

$$
A_{\varepsilon} \eta=K(x) f(|\eta|) \eta+W(x)|\eta|^{2^{*}-2} \eta .
$$

In what follows, we write $F(|z|):=\int_{0}^{|z|} f(s) s d s$. Before stating our results, we make the following assumptions for the nonlinearity:

$\left(\mathrm{F}_{0}\right) f \in C^{1}\left(\mathbb{R}^{+}, \mathbb{R}^{+}\right), f(0)=0$ and $f^{\prime}(s) \geq 0$ for $s>0$, where $\mathbb{R}^{+}=[0, \infty)$;

$\left(\mathrm{F}_{1}\right)$ there exist $c>0$ and $p \in\left(2,2^{*}\right)$ such that $f(s) \leq c\left(1+s^{p-2}\right)$ for $s \geq 0$;

$\left(\mathrm{F}_{2}\right)$ there exist $\theta>2,2<\sigma \leq p$ and $c_{0}>0$ such that $c_{0} s^{\sigma} \leq F(s) \leq$ $f(s) s^{2} / \theta$ for all $s \neq 0$.

A typical example is the power function $f(s)=s^{\sigma-2}$. For describing our study we first need to present some information of the superlinear and subcritical 
system

$$
\begin{cases}-\Delta \psi+b \cdot \nabla \psi+\psi=|\eta|^{\sigma-2} \varphi & \text { in } \mathbb{R}^{N}, \\ -\Delta \varphi-b \cdot \nabla \varphi+\varphi=|\eta|^{\sigma-2} \psi & \text { in } \mathbb{R}^{N},\end{cases}
$$

where $\sigma$ is the constant from $\left(\mathrm{F}_{2}\right)$. Let $\gamma$ denote the least energy of the ground state solution of system (1.5), it follows from the conclusion of [42] that $\gamma>0$ is attained. Furthermore, set

$$
\mathscr{R}_{\sigma}:=\left(\frac{S^{N / 2} c_{0}^{2 /(\sigma-2)}}{N \gamma}\right)^{(\sigma-2) / 2}
$$

and

$$
\kappa:=\max _{x \in \mathbb{R}^{N}} K(x), \quad \kappa_{\infty}:=\limsup _{|x| \rightarrow \infty} K(x), \quad \omega:=\max _{x \in \mathbb{R}^{N}} W(x),
$$

where $c_{0}$ is given in $\left(F_{2}\right)$ and $S$ denotes the best Sobolev embedding constant

$$
S\left(\int_{\mathbb{R}^{N}}|z|^{2^{*}}\right)^{2 / 2^{*}} \leq \int_{\mathbb{R}^{N}}|\nabla z|^{2}, \quad \text { for all } z \in \mathcal{D}^{1,2}\left(\mathbb{R}^{N}, \mathbb{R}^{2}\right) .
$$

For the nonlinear potentials $K$ and $W$, we suppose that

$\left(\mathrm{K}_{0}\right) K, W \in C\left(\mathbb{R}^{N}\right)$ with $a:=\inf _{x \in \mathbb{R}^{N}} K(x)>0, \inf _{x \in \mathbb{R}^{N}} W(x)>0$ and $\kappa_{\infty}<\kappa$, $\lim \sup W(x) \leq \omega ;$

$$
|x| \rightarrow \infty
$$

$\left(\mathrm{K}_{1}\right) \kappa_{\infty}^{-1} \cdot \omega^{(N-2)(\sigma-2) / 4}<\mathscr{R}_{\sigma}$

For showing the concentration phenomenon, we define the following concentration set $\mathscr{M}:=\left\{x \in \mathbb{R}^{N}: K(x)=\kappa, W(x)=\omega\right\}$. Without loss of generality, below we may assume $0 \in \mathscr{M}$ throughout the paper. Now, we are ready to state the main result of this paper.

Theorem 1.1. Assume that $|b| \leq 2,\left(\mathrm{~F}_{0}\right)-\left(\mathrm{F}_{2}\right),\left(\mathrm{K}_{0}\right)$ and $\left(\mathrm{K}_{1}\right)$ are satisfied. Then, for all sufficiently small $\varepsilon>0$,

(a) $\left(\mathcal{P}_{\varepsilon}\right)$ has a ground state solution $\eta_{\varepsilon}$;

(b) $\mathscr{L}_{\varepsilon}$ is compact in $H^{2}\left(\mathbb{R}^{N}\right)$, where $\mathscr{L}_{\varepsilon}$ denotes the set of all ground state solutions of $\left(\mathcal{P}_{\varepsilon}\right)$;

(c) there exists a maximum point $x_{\varepsilon}$ of $\left|\eta_{\varepsilon}(x)\right|$ with $\lim _{\varepsilon \rightarrow 0} \operatorname{dist}\left(x_{\varepsilon}, \mathscr{M}\right)=0$, and setting $w_{\varepsilon}(x):=\eta_{\varepsilon}\left(\varepsilon x+x_{\varepsilon}\right)$, $v_{\varepsilon}$ converges in $H^{2}\left(\mathbb{R}^{N}\right)$ to a ground state solution of

$$
\begin{cases}-\Delta \psi+b \cdot \nabla \psi+\psi=\kappa f(|\eta|) \varphi+\omega|\eta|^{2^{*}-2} \varphi & \text { in } \mathbb{R}^{N}, \\ -\Delta \varphi-b \cdot \nabla \varphi+\varphi=\kappa f(|\eta|) \psi+\omega|\eta|^{2^{*}-2} \psi & \text { in } \mathbb{R}^{N},\end{cases}
$$

(d) there exist $c, C>0$ such that $\left|\eta_{\varepsilon}(x)\right| \leq C \exp \left(-c\left|x-x_{\varepsilon}\right| / \varepsilon\right)$. 
For the proof of our results, we do not handle system $\left(\mathcal{P}_{\varepsilon}\right)$ directly, but instead we handle an equivalent system to $\left(\mathcal{P}_{\varepsilon}\right)$. For this purpose, set $z(x)=$ $(u(x), v(x))=(\psi(\varepsilon x), \varphi(\varepsilon x))=\eta(\varepsilon x), K_{\varepsilon}(x)=K(\varepsilon x)$ and $W_{\varepsilon}(x)=W(\varepsilon x)$. Then system $\left(\mathcal{P}_{\varepsilon}\right)$ is equivalent to the following:

$$
\begin{cases}-\Delta u+b \cdot \nabla u+u=K_{\varepsilon}(x) f(|z|) v+W_{\varepsilon}(x)|z|^{2^{*}-2} v & \text { in } \mathbb{R}^{N} \\ -\Delta v-b \cdot \nabla v+v=K_{\varepsilon}(x) f(|z|) u+W_{\varepsilon}(x)|z|^{2^{*}-2} u & \text { in } \mathbb{R}^{N} .\end{cases}
$$

Moreover, system $\left(\mathcal{P}_{\varepsilon}^{\prime}\right)$ can be expressed as

$$
A z=K_{\varepsilon}(x) f(|z|) z+W_{\varepsilon}(x)|z|^{2^{*}-2} z,
$$

where

$$
A=\left(\begin{array}{cc}
0 & -\Delta-b \cdot \nabla+1 \\
-\Delta+b \cdot \nabla+1 & 0
\end{array}\right) .
$$

Clearly, (1.4) is equivalent to (1.6). We will, in the sequel, focus on this equivalent problem.

As a motivation we recall that there is a large number of investigations devoted to the study on the semiclassical states of Schrödinger equations

$$
-\varepsilon^{2} \Delta u+V(x) u=f(x, u), \quad u \in H^{1}\left(\mathbb{R}^{N}\right) .
$$

It was shown, under suitable assumptions of course, that for all $\varepsilon>0,(1.7)$ possesses a ground state $u_{\varepsilon}$ which concentrates on the set of minimum points of $V$ as $\varepsilon \rightarrow 0$. For example, see [2], [7], [8], [11], [17], [18], [21], [23], [27], [28], [31]. Note that, since the Schrödinger operator $-\Delta+V$ is bounded from below, techniques based on the mountain pass theorem are well applied to the investigation. However, for our problem, the mountain pass structure no longer applies since the corresponding energy functional is strongly indefinite, the classical critical point theory cannot be applied directly. Hence our problem poses more challenges in the calculus of variation in nature.

Our argument is variational, the semiclassical solutions are obtained as critical points of the energy functional $\Phi_{\varepsilon}$ associated to $\left(\mathcal{P}_{\varepsilon}\right)$. But one of the main technical difficulties to be overcome is that the energy functional $\Phi_{\varepsilon}$ is strongly indefinite. Inspired by Ackermann [1], we utilize a reduced method, which reduces the strongly indefinite case to the mountain pass case. Hence, we construct a reduced functional $I_{\varepsilon}$ whose critical points are in one-to-one correspondence to critical points of $\Phi_{\varepsilon}$. With the help of the Nehari manifold $\mathscr{N}_{\varepsilon}$ of $I_{\varepsilon}$, an important information of the least energy $c_{\varepsilon}$ will be obtained. Moreover, by applying some ideas used in [13], [15], we prove that the value $c_{\varepsilon}$ is attained. In addition, we also prove the concentration phenomenon of semiclassical solutions. Finally, for proving the exponential decay, the Kato inequality seems not work well due to the presence of the gradient term in system $\left(\mathcal{P}_{\varepsilon}\right)$, we handle, instead of $\Delta|z|$ 
in the Kato inequality, the square of $\Delta|z|$, that is $\Delta|z|^{2}$, and then describe the decay at infinity in a subtle way.

\section{Variational setting and linking structure}

Below by $|\cdot|_{q}$ we denote the usual $L^{q}$-norm, $(\cdot, \cdot)_{2}$ denotes the usual $L^{2}$ inner product, $c, c_{i}$ or $C_{i}$ stand for different positive constants. Denote by $\sigma(A)$ and $\sigma_{e}(A)$ the spectrum and the essential spectrum of the operator $A$, respectively. In order to establish suitable variational framework for system $\left(\mathcal{P}_{\varepsilon}^{\prime}\right)$, we make use of the following lemmas, which are two special cases in [39].

Lemma 2.1. ([39, Lemma 2.1]) The operator $A$ is a selfadjoint operator on $L^{2}\left(\mathbb{R}^{N}, \mathbb{R}^{2}\right)$ with domain $\mathcal{D}(A):=H^{2}\left(\mathbb{R}^{N}, \mathbb{R}^{2}\right)$.

Lemma 2.2. ([39, Lemma 2.3]) The following two conclusions hold:

(a) $\sigma(A)=\sigma_{e}(A)$, i.e. A has only essential spectrum;

(b) $\sigma(A) \subset \mathbb{R} \backslash(-1,1)$ and $\sigma(A)$ is symmetric with respect to origin.

It follows from Lemmas 2.1 and 2.2 that the space $L^{2}:=L^{2}\left(\mathbb{R}^{N}, \mathbb{R}^{2}\right)$ possesses the orthogonal decomposition

$$
L^{2}=L^{-} \oplus L^{+}, \quad z=z^{-}+z^{+}
$$

such that $A$ is negative definite (resp. positive definite) in $L^{-}$(resp. $L^{+}$). Let $|A|$ denote the absolute value of $A$ and $|A|^{1 / 2}$ be the square root of $|A|$. Let $E=\mathcal{D}\left(|A|^{1 / 2}\right)$ be the Hilbert space with the inner product

$$
\langle z, w\rangle=\left(|A|^{1 / 2} z,|A|^{1 / 2} w\right)_{2}
$$

and norm $\|z\|=\langle z, z\rangle^{1 / 2}$. There is an induced decomposition

$$
E=E^{-} \oplus E^{+}, \quad \text { where } E^{ \pm}=E \cap L^{ \pm},
$$

which is orthogonal with respect to the inner products $(\cdot, \cdot)_{2}$ and $\langle\cdot, \cdot\rangle$.

Lemma 2.3. ([39, Lemma 2.4]) $\|\cdot\|$ and $\|\cdot\|_{H^{1}}$ are equivalent norms. Therefore, $E$ embeds continuously into $L^{p}:=L^{p}\left(\mathbb{R}^{N}, \mathbb{R}^{2}\right)$ for any $p \in\left[2,2^{*}\right]$ and compactly into $L_{\mathrm{loc}}^{p}:=L_{\mathrm{loc}}^{p}\left(\mathbb{R}^{N}, \mathbb{R}^{2}\right)$ for any $p \in\left[2,2^{*}\right)$, and there exists a constant $\pi_{p}$ such that

$$
\pi_{p}|z|_{p} \leq\|z\|, \quad \text { for all } z \in E, p \in\left[2,2^{*}\right] .
$$

In virtue of assumptions $\left(\mathrm{F}_{0}\right)-\left(\mathrm{F}_{2}\right)$, for any $\varepsilon>0$, there exist positive constants $r_{\varepsilon}, C_{\varepsilon}$ and $C_{\varepsilon}^{\prime}$ such that

$$
\begin{cases}f(s) \leq \varepsilon & \text { for all } 0 \leq s \leq r_{\varepsilon} \\ F(|z|) \leq \varepsilon|z|^{2}+C_{\varepsilon}|z|^{p} & \text { for all } z \in \mathbb{R}^{2}, p \in\left(2,2^{*}\right), \\ F(|z|) \geq C_{\varepsilon}^{\prime}|z|^{\theta}-\varepsilon|z|^{2} & \text { for all } z \in \mathbb{R}^{2}, \theta>2\end{cases}
$$


On $E$ we define the following functional:

$$
\Phi_{\varepsilon}(z)=\frac{1}{2}\left(\left\|z^{+}\right\|^{2}-\left\|z^{-}\right\|^{2}\right)-\int_{\mathbb{R}^{N}} K_{\varepsilon}(x) F(|z|)-\frac{1}{2^{*}} \int_{\mathbb{R}^{N}} W_{\varepsilon}(x)|z|^{2^{*}} .
$$

Lemma 2.2 implies that $\Phi_{\varepsilon}$ is strongly indefinite. Moreover, our hypotheses imply that $\Phi_{\varepsilon} \in C^{1}(E, \mathbb{R})$, and a standard argument shows that critical points of $\Phi_{\varepsilon}$ are solutions of problem $\left(\mathcal{P}_{\varepsilon}^{\prime}\right)$ (see [10], [46]). For convenience, let

$$
\Psi_{\varepsilon}(z)=\int_{\mathbb{R}^{N}} K_{\varepsilon}(x) F(|z|)+\frac{1}{2^{*}} \int_{\mathbb{R}^{N}} W_{\varepsilon}(x)|z|^{2^{*}} .
$$

LEMMA 2.4. $\Psi_{\varepsilon}$ is weakly sequentially lower semi-continuous. $\Psi_{\varepsilon}^{\prime}$ is weakly sequentially continuous.

Using the Lemma 2.3, one can check easily the above lemma, here we omit the details (see, for example [10], [22] and [46]).

Set, for $r>0, B_{r}^{+}=\left\{z \in E^{+}:\|z\| \leq r\right\}$ and $S_{r}^{+}=\left\{z \in E^{+}:\|z\|=r\right\}$, and for $e \in E^{+}, E_{e}:=E^{-} \oplus \mathbb{R}^{+} e$ with $\mathbb{R}^{+}=[0, \infty)$. Now we discuss the linking structure of $\Phi_{\varepsilon}$.

LEMma 2.5. There exist $r>0$ and $\rho>0$ both independent of $\varepsilon$ such that $\left.\Phi_{\varepsilon}\right|_{B_{r}^{+}}(z) \geq 0$ and $\left.\Phi_{\varepsilon}\right|_{S_{r}^{+}}(z) \geq \rho$.

Proof. Observe that, $\pi_{2}=1$ by Lemma $2.2(\mathrm{~b})$. For any $z \in E^{+}$, by $(2.1)$ and (2.2) we have

$$
\begin{aligned}
\Phi_{\varepsilon}(z) & =\frac{1}{2}\|z\|^{2}-\int_{\mathbb{R}^{N}} K_{\varepsilon}(x) F(|z|)-\frac{1}{2^{*}} \int_{\mathbb{R}^{N}} W_{\varepsilon}(x)|z|^{2^{*}} \\
& \geq \frac{1}{2}\|z\|^{2}-\varepsilon \kappa|z|_{2}^{2}-C_{\varepsilon} \kappa|z|_{p}^{p}-\frac{\omega}{2^{*}}|z|_{2^{*}}^{2^{*}} \\
& \geq \frac{1}{2}\|z\|^{2}-\varepsilon \kappa\|z\|^{2}-C_{\varepsilon} \kappa \pi_{p}^{-p}\|z\|^{p}-\frac{\omega}{2^{*}} \pi_{2^{*}}^{-2^{*}}\|z\|^{2^{*}} \\
& =\left(\frac{1}{2}-\varepsilon \kappa\right)\|z\|^{2}-C_{\varepsilon} \kappa \pi_{p}^{-p}\|z\|^{p}-\frac{\omega}{2^{*}} \pi_{2^{*}}^{-2^{*}}\|z\|^{2^{*}}
\end{aligned}
$$

Since $p \in\left(2,2^{*}\right)$, choosing suitable $\varepsilon, r>0$ we see that the desired conclusion holds.

Lemma 2.6. For any $e \in E^{+} \backslash\{0\}$, there exist $R_{e}>0$ and $C=C_{e}>0$ both independent of $\varepsilon$ such that $\Phi_{\varepsilon}(z)<0$ for all $z \in E_{e} \backslash B_{R_{e}}$ and $\max \Phi_{\varepsilon}\left(E_{e}\right) \leq C$.

Proof. For any $z \in E_{e}$, that is $z=t e+v$ for some $t \geq 0$ and $v \in E^{-}$. By the Young inequality and (2.2), we have

$$
\begin{aligned}
\Phi_{\varepsilon}(z) & =\frac{1}{2}\left(t^{2}\|e\|^{2}-\|v\|^{2}\right)-\int_{\mathbb{R}^{N}} K_{\varepsilon}(x) F(|t e+v|)-\frac{1}{2^{*}} \int_{\mathbb{R}^{N}} W_{\varepsilon}(x)|t e+v|^{2^{*}} \\
& \leq \frac{1}{2}\left(t^{2}\|e\|^{2}-\|v\|^{2}\right)+a \varepsilon|t e+v|_{2}^{2}-a C_{\varepsilon}^{\prime}|t e+v|_{\theta}^{\theta}
\end{aligned}
$$




$$
\begin{aligned}
\leq & t^{2}\left(\frac{1}{2}\|e\|^{2}+a \varepsilon|e|_{2}^{2}\right)+\left(a \varepsilon|v|_{2}^{2}-\frac{1}{2}\|v\|^{2}\right)-a C_{\varepsilon}^{\prime}|t e+v|_{\theta}^{\theta} \\
\leq & t^{2}\left(\frac{1}{2}\|e\|^{2}+a \varepsilon|e|_{2}^{2}\right)+\left(a \varepsilon|v|_{2}^{2}-\frac{1}{2}\|v\|^{2}\right) \\
& -t^{\theta} a C_{\varepsilon}^{\prime}(1-(\theta-1) \varsigma)|e|_{\theta}^{\theta}+\varsigma^{-(\theta-1)} a C_{\varepsilon}^{\prime}|v|_{\theta}^{\theta}
\end{aligned}
$$

for some $0<\varsigma<1 /(\theta-1)$. Since $\theta>2$, choosing large $R_{e}>0$ we see that the desired conclusion holds.

Similar to an argument in [30], we define the following minimax value:

$$
c_{\varepsilon}:=\inf _{e \in E^{+} \backslash\{0\}} \max _{z \in E_{e}} \Phi_{\varepsilon}(z) .
$$

As a consequence of Lemmas 2.5 and 2.6, we have

Lemma 2.7. There is $C>0$ independent of $\varepsilon$ such that $\rho \leq c_{\varepsilon}<C$.

Recall that a sequence $\left\{z_{n}\right\} \subset E$ is said to be a $(\mathrm{PS})_{c}$ sequence for a functional $\Phi \in C^{1}(E, \mathbb{R})$ if $\Phi\left(z_{n}\right) \rightarrow c$ and $\Phi^{\prime}\left(z_{n}\right) \rightarrow 0$, and $\Phi$ is said to satisfy the $(\mathrm{PS})_{c}$ condition if any $(\mathrm{PS})_{c}$ sequence for $\Phi$ has a convergent subsequence. With Lemmas $2.4,2.5$ and 2.6 and by a standard linking argument it follows that $\Phi_{\varepsilon}$ has a $(\mathrm{PS})_{c_{\varepsilon}}$ sequence (see [10] and [30]). Obviously, if $\Phi_{\varepsilon}$ satisfies the $(\mathrm{PS})_{c_{\varepsilon}}$ condition, then $c_{\varepsilon}$ is a critical value of $\Phi_{\varepsilon}$. Unfortunately, since there is no compactly embedding from $H^{1}$ into $L^{p}$ for $2 \leq p<2^{*}$, then the $(\mathrm{PS})_{c_{\varepsilon}}$ condition does not hold in general, we have to go through more analysis.

Following Ackermann [1], for a fixed $z \in E^{+}$we introduce $\phi_{\varepsilon, z}: E^{-} \rightarrow \mathbb{R}$ defined by

$$
\phi_{\varepsilon, z}(w)=\Phi_{\varepsilon}(z+w) .
$$

A direct computation gives, for any $w, \varphi \in E^{-}$,

$$
\begin{aligned}
\phi_{\varepsilon, z}^{\prime \prime}(w)[\varphi, \varphi]= & -\|\varphi\|^{2}-\Psi_{\varepsilon}^{\prime \prime}(z+w)[\varphi, \varphi] \\
\leq & -\|\varphi\|^{2}-\left(2^{*}-1\right) \int_{\mathbb{R}^{N}} W_{\varepsilon}(x)|z+w|^{2^{*}-2}|\varphi|^{2} \\
& -\int_{\mathbb{R}^{N}} K_{\varepsilon}(x)\left(f^{\prime}(|z+w|)|z+w \| \varphi|^{2}+f(|z+w|)|\varphi|^{2}\right) .
\end{aligned}
$$

By $\left(\mathrm{K}_{0}\right)$ and $\left(\mathrm{F}_{0}\right)$, it is easy to see that $\phi_{\varepsilon, z}^{\prime \prime}(w)[\varphi, \varphi]<0$ for $\varphi \neq 0$. In addition,

$$
\phi_{\varepsilon, z}(w) \leq \frac{1}{2}\|z\|^{2}-\frac{1}{2}\|w\|^{2} \rightarrow-\infty \quad \text { as }\|w\| \rightarrow \infty .
$$

Therefore, there is a unique $h_{\varepsilon}(z) \in E^{-}$such that $\phi_{\varepsilon, z}\left(h_{\varepsilon}(z)\right)=\max _{w \in E^{-}} \phi_{\varepsilon, z}(w)$ and $w \neq h_{\varepsilon}(z)$ if and only if $\Phi_{\varepsilon}(z+w)<\Phi_{\varepsilon}\left(z+h_{\varepsilon}(z)\right)$.

It is clear that $0=\phi_{\varepsilon, z}^{\prime}\left(h_{\varepsilon}(z)\right) w$ for all $w \in E^{-}$. For any $z \in E^{+}$and $w \in E^{-}$, setting $v=w-h_{\varepsilon}(z)$ and $g(t)=\phi_{\varepsilon, z}\left(h_{\varepsilon}(z)+t v\right)$, one has $g(1)=\phi_{\varepsilon, z}(w)$, 
$g(0)=\phi_{\varepsilon, z}\left(h_{\varepsilon}(z)\right)$ and $g^{\prime}(0)=0$. Thus

$$
g(1)-g(0)=\int_{0}^{1}(1-t) g^{\prime \prime}(t) d t .
$$

This implies that

$$
\begin{aligned}
\phi_{\varepsilon, z}(w)-\phi_{\varepsilon, z}\left(h_{\varepsilon}(z)\right)=\int_{0}^{1}(1-t) \phi_{\varepsilon, z}^{\prime \prime}\left(h_{\varepsilon}(z)+t v\right)[v, v] d t \\
=-\int_{0}^{1}(1-t)\left[\|v\|^{2}+\left(2^{*}-1\right) \int_{\mathbb{R}^{N}} W_{\varepsilon}(x)\left|z+h_{\varepsilon}(z)+t v\right|^{2^{*}-2}|v|^{2}\right. \\
\quad+\int_{\mathbb{R}^{N}} K_{\varepsilon}(x)\left(f^{\prime}\left(\left|z+h_{\varepsilon}(z)+t v\right|\right) \frac{\left|\left(z+h_{\varepsilon}(z)+t v\right) v\right|^{2}}{\left|z+h_{\varepsilon}(z)+t v\right|}\right. \\
\left.\left.\quad+f\left(\left|z+h_{\varepsilon}(z)+t v\right|\right)|v|^{2}\right)\right] d t \\
=-\frac{1}{2}\|v\|^{2}-\int_{\mathbb{R}^{N}} H_{\varepsilon}(x),
\end{aligned}
$$

where

$$
\begin{aligned}
H_{\varepsilon}(x) & =\int_{0}^{1}(1-t)\left(K _ { \varepsilon } ( x ) \left[f^{\prime}\left(\left|z+h_{\varepsilon}(z)+t v\right|\right) \frac{\left|\left(z+h_{\varepsilon}(z)+t v\right) v\right|^{2}}{\left|z+h_{\varepsilon}(z)+t v\right|}\right.\right. \\
& \left.\left.+f\left(\left|z+h_{\varepsilon}(z)+t v\right|\right)|v|^{2}\right]+\left(2^{*}-1\right) W_{\varepsilon}(x)\left|z+h_{\varepsilon}(z)+t v\right|^{2^{*}-2}|v|^{2}\right) d t .
\end{aligned}
$$

Hence

$$
\Phi_{\varepsilon}\left(z+h_{\varepsilon}(z)\right)-\Phi_{\varepsilon}(z+w)=\frac{1}{2}\|v\|^{2}+\int_{\mathbb{R}^{N}} H_{\varepsilon}(x) .
$$

Now, define the reduced functional $I_{\varepsilon}: E^{+} \rightarrow \mathbb{R}$ by

$$
I_{\varepsilon}(z)=\Phi_{\varepsilon}\left(z+h_{\varepsilon}(z)\right)=\frac{1}{2}\left(\|z\|^{2}-\left\|h_{\varepsilon}(z)\right\|^{2}\right)-\Psi_{\varepsilon}\left(z+h_{\varepsilon}(z)\right),
$$

and its Nehari manifold by $\mathscr{N}_{\varepsilon}:=\left\{z \in E^{+} \backslash\{0\}: I_{\varepsilon}^{\prime}(z) z=0\right\}$. Then critical points of $I_{\varepsilon}$ and $\Phi_{\varepsilon}$ are in one-to-one correspondence via the injective map $z \mapsto$ $z+h_{\varepsilon}(z)$ from $E^{+}$into $E$.

Lemma 2.8. For any $z \in E^{+} \backslash\{0\}$, there is a unique $t=t(z)>0$ such that $t(z) z \in \mathscr{N}_{\varepsilon}$.

For the proof of the previous lemma, we refer to $[1,14]$. Here we omit the details.

Lemma 2.9. We define $d_{\varepsilon}:=\inf _{z \in \mathscr{N}_{\varepsilon}} I_{\varepsilon}(z)$, and $d_{\varepsilon}=c_{\varepsilon}$.

Proof. Indeed, given $e \in E^{+}$, if $z=w+s e \in E_{e}$ with $\Phi_{\varepsilon}(z)=\max _{v \in E_{e}} \Phi_{\varepsilon}(v)$ then the restriction $\left.\Phi_{\varepsilon}\right|_{E_{e}}$ of $\Phi_{\varepsilon}$ on $E_{e}$ satisfies $\left(\left.\Phi_{\varepsilon}\right|_{E_{e}}\right)^{\prime}(z)=0$ which implies $w=h_{\varepsilon}(s e)$ and $I_{\varepsilon}^{\prime}(s e)(s e)=\Phi_{\varepsilon}^{\prime}(z)(s e)=0$, i.e. $s e \in \mathscr{N}_{\varepsilon}$. Thus $d_{\varepsilon} \leq c_{\varepsilon}$. On the 
other hand, if $z \in \mathscr{N}_{\varepsilon}$ then $\left(\left.\Phi_{\varepsilon}\right|_{E_{z}}\right)^{\prime}\left(z+h_{\varepsilon}(z)\right)=0$ so $c_{\varepsilon} \leq \max _{v \in E_{z}} \Phi_{\varepsilon}(v)=I_{\varepsilon}(z)$. Thus $d_{\varepsilon} \geq c_{\varepsilon}$. This proves $d_{\varepsilon}=c_{\varepsilon}$.

Lemma 2.10. For any $e \in E^{+} \backslash\{0\}$, there exists $T_{e}>0$ independent of $\varepsilon>0$ such that $t_{\varepsilon} \leq T_{e}$ for $t_{\varepsilon}>0$ satisfying $t_{\varepsilon} e \in \mathscr{N}_{\varepsilon}$.

Proof. Since $I_{\varepsilon}^{\prime}\left(t_{\varepsilon} e\right)\left(t_{\varepsilon} e\right)=0$, one gets

$$
\Phi_{\varepsilon}\left(t_{\varepsilon} e+h_{\varepsilon}\left(t_{\varepsilon} e\right)\right)=\max _{z \in E_{e}} \Phi_{\varepsilon}(z) .
$$

This, together with Lemmas 2.6 and 2.9, implies that

$$
c_{\varepsilon} \leq \Phi_{\varepsilon}\left(t_{\varepsilon} e+h_{\varepsilon}\left(t_{\varepsilon} e\right)\right) \leq c_{1} t_{\varepsilon}^{2}-c_{2} t_{\varepsilon}^{\theta}+c_{3},
$$

from which one can show the desired conclusion.

Let $\mathscr{K}_{\varepsilon}:=\left\{z \in E \backslash\{0\}: \Phi_{\varepsilon}^{\prime}(z)=0\right\}$ be the critical set of $\Phi_{\varepsilon}$. Since critical points of $I_{\varepsilon}$ and $\Phi_{\varepsilon}$ are in one-to-one correspondence via the injective map $z \mapsto z+h_{\varepsilon}(z)$ from $E^{+}$into $E$, from Lemma 2.9, it is easy to see that if $\mathscr{K}_{\varepsilon} \neq \emptyset$ then $c_{\varepsilon}=\inf \left\{\Phi_{\varepsilon}(z): z \in \mathscr{K}_{\varepsilon}\right\}$. Using the standard bootstrap argument (see e.g. [14], [16] for the iterative steps), one obtains easily the following result.

Lemma 2.11. If $z \in \mathscr{K}_{\varepsilon}$ with $\left|\Phi_{\varepsilon}(z)\right| \leq C_{1}$ and $|u|_{2} \leq C_{2}$, then, for any $r \geq 2, z \in H^{2, r}$ and $\|z\|_{H^{2, r}} \leq \Lambda_{r}$, where $\Lambda_{r}$ depends only on $C_{1}, C_{2}$ and $r$.

Let $\mathscr{L}_{\varepsilon}$ be the set of all least energy solutions of $\Phi_{\varepsilon}$. If $z \in \mathscr{L}_{\varepsilon}$ then $\Phi_{\varepsilon}(z)=c_{\varepsilon}$ and a standard argument shows that $\mathscr{L}_{\varepsilon}$ is bounded in $E$, hence, $|z|_{2} \leq C_{2}$ for $z \in \mathscr{L}_{\varepsilon}$, some $C_{2}>0$ independent of $\varepsilon$. Therefore, as a consequence of Lemmas 2.9 and 2.11 we see that, for each $r \geq 2$, there is $C_{r}>0$ independent of $\varepsilon$ such that $\|z\|_{H^{2, r}} \leq C_{r}$ for all $z \in \mathscr{L}_{\varepsilon}$. This, together with the Sobolev embedding theorem, implies that there is $C_{\infty}>0$ independent of $\varepsilon$ with

$$
|z|_{\infty} \leq C_{\infty} \text { for all } z \in \mathscr{L}_{\varepsilon} .
$$

Lemma 2.12. Assume that $|b| \leq 2$, there is $C_{0}>0$ independent of $x, z \in \mathscr{L}_{\varepsilon}$ and $\varepsilon>0$ such that

$$
|z(x)| \leq C_{0}\left(\int_{B_{1}(x)}|z(y)|^{2} d y\right)^{1 / 2}, \quad x \in \mathbb{R}^{N}, z \in \mathscr{L}_{\varepsilon},
$$

where $B_{1}(x)=\{y:|y-x| \leq 1\}$.

Proof. Let $z=(u, v)$ be a solution of $\left(\mathcal{P}_{\varepsilon}^{\prime}\right)$. Recall that $|z|^{2}=u^{2}+v^{2}$. Then, for $i=1, \ldots, N$,

$$
\begin{aligned}
D_{i}\left(|z|^{2}\right) & =2|z| D_{i}(|z|)=2|z| \frac{u D_{i} u+v D_{i} v}{|z|}=2\left(u D_{i} u+v D_{i} v\right), \\
D_{i i}\left(|z|^{2}\right) & =2 D_{i}\left(u D_{i} u+v D_{i} v\right)=2\left(D_{i} u D_{i} u+u D_{i i} u+D_{i} v D_{i} v+v D_{i i} v\right) .
\end{aligned}
$$


This yields that

$$
\begin{aligned}
\Delta|z|^{2} & =\sum_{i=1}^{N} D_{i i}\left(|z|^{2}\right)=2 \sum_{i=1}^{N}\left(D_{i} u D_{i} u+u D_{i i} u+D_{i} v D_{i} v+v D_{i i} v\right) \\
& =2\left(|\nabla u|^{2}+|\nabla v|^{2}+u \Delta u+v \Delta v\right)=2\left(|\nabla z|^{2}+z \Delta z\right),
\end{aligned}
$$

where $\nabla z=(\nabla u, \nabla v)$. Since $z=(u, v)$ is a solution of $\left(\mathcal{P}_{\varepsilon}^{\prime}\right)$, then

$$
\begin{array}{rl}
\Delta|z|^{2}= & 2\left(|\nabla z|^{2}+z \Delta z\right) \\
= & 2\left(|u|^{2}+|v|^{2}-2 W_{\varepsilon}(x)|z|^{2^{*}-2} u v\right. \\
& \left.-2 K_{\varepsilon}(x) f(|z|) u v+b \cdot \nabla u u-b \cdot \nabla v v+|\nabla z|^{2}\right) \\
\geq 2 & 2\left(|z|^{2}-\omega|z|^{2^{*}-2}\left(|u|^{2}+|v|^{2}\right)\right. \\
& \left.-\kappa f(|z|)\left(|u|^{2}+|v|^{2}\right)+b \cdot \nabla u u-b \cdot \nabla v v+|\nabla z|^{2}\right) \\
\geq 2 & 2\left(|z|^{2}-\omega|z|^{2^{*}}-\kappa f(|z|)|z|^{2}\right. \\
& \left.\left.-|b|^{(} \frac{|\nabla u|^{2}+|u|^{2}}{2}+\frac{|\nabla v|^{2}+|v|^{2}}{2}\right)+|\nabla z|^{2}\right) \\
= & 2\left(|z|^{2}-\omega|z|^{2^{*}}-\kappa f(|z|)|z|^{2}-\frac{|b|}{2}|z|^{2}-\frac{|b|}{2}|\nabla z|^{2}+|\nabla z|^{2}\right)^{\prime} \\
\geq 2 & 2\left(|z|^{2}-\omega|z|^{2^{*}}-\kappa f(|z|)|z|^{2}-\frac{|b|}{2}|z|^{2}\right)
\end{array}
$$

as $|b| \leq 2$. On the other hand, by some similar arguments as in [9], we know that $|z(x)| \rightarrow 0$ as $|x| \rightarrow \infty$. Thus, for any $\alpha>0$, it follows from $\left(F_{1}\right)$ that there is $R>0$ such that

$$
f(|z|) \leq \alpha, \quad|x| \geq R
$$

Therefore, by (2.8) and (2.9), there exists $\delta>0$ such that

$$
\Delta|z|^{2} \geq-\delta|z|^{2}, \quad x \in \mathbb{R}^{N}
$$

which implies that $|z|^{2}$ is a sub-solution of the equation $(-\Delta-\delta) z=0$. Moreover, by the sub-solution estimate [19], we have

$$
|z(x)| \leq C_{0}\left(\int_{B_{1}(x)}|z(y)|^{2} d y\right)^{1 / 2}, \quad x \in \mathbb{R}^{N},
$$

with $C_{0}>0$ independent of $x, z \in \mathscr{L}_{\varepsilon}$ and $\varepsilon>0$. 


\section{The autonomous problems}

In order to prove our main result, we need some results on related autonomous system. For the constants $\mu, \nu>0$, consider the subcritical autonomous system

$$
\begin{cases}-\Delta u+b \cdot \nabla u+u=\mu f(|z|) v & \text { in } \mathbb{R}^{N}, \\ -\Delta v-b \cdot \nabla v+v=\mu f(|z|) u & \text { in } \mathbb{R}^{N}\end{cases}
$$

and the critical system

$$
\begin{cases}-\Delta u+b \cdot \nabla u+u=\mu f(|z|) v+\nu|z|^{2^{*}-2} v & \text { in } \mathbb{R}^{N} \\ -\Delta v-b \cdot \nabla v+v=\mu f(|z|) u+\nu|z|^{2^{*}-2} u & \text { in } \mathbb{R}^{N}\end{cases}
$$

Firstly, we introduce the results of the subcritical system. It is well known that the solutions of system (3.1) are critical points of the functional

$$
\Phi_{\mu}(z)=\frac{1}{2}\left(\left\|z^{+}\right\|^{2}-\left\|z^{-}\right\|^{2}\right)-\mu \int_{\mathbb{R}^{N}} F(|z|):=\frac{1}{2}\left(\left\|z^{+}\right\|^{2}-\left\|z^{-}\right\|^{2}\right)-\Psi_{\mu}(z)
$$

defined for $z=z^{-}+z^{+} \in E=E^{-} \oplus E^{+}$. Denote the critical set, the least energy, and the set of ground state solutions of $\Phi_{\mu}$ as follows:

$$
\begin{aligned}
\mathscr{K}_{\mu} & :=\left\{z \in E: \Phi_{\mu}^{\prime}(z)=0\right\}, \\
c_{\mu} & :=\inf \left\{\Phi_{\mu}(z): z \in \mathscr{K}_{\mu} \backslash\{0\}\right\}, \\
\mathscr{L}_{\mu} & :=\left\{z \in \mathscr{K}_{\mu}: \Phi_{\mu}(z)=c_{\mu}\right\} .
\end{aligned}
$$

LEMMA 3.1. There hold the following conclusions:

(a) system (3.1) has at least one ground state solution, i.e., $\mathscr{K}_{\mu} \neq \emptyset$, and $c_{\mu}>0$

(b) $c_{\mu}$ is attained, and $\mathscr{L}_{\mu}$ is compact in $H^{2}\left(\mathbb{R}^{N}\right)$;

(c) there exist $C, c>0$ such that $|z(x)| \leq C \exp (-c|x|)$, for $x \in \mathbb{R}^{N}$ and $z \in \mathscr{L}_{\mu}$.

Proof. For the proof of (a) and (b), we refer to the proof in [34, Theorem 1.1]. Now, we show that (c) holds. Let $z=(u, v) \in \mathscr{L}_{\mu}$. By (b), $|z(x)| \rightarrow 0$ as $|x| \rightarrow \infty$ uniformly in $z \in \mathscr{L}_{\mu}$. In fact, if not, then there exist $\varrho>0$, $\left\{z_{j}\right\} \subset \mathscr{L}_{\mu}$ and $\left\{x_{j}\right\} \subset \mathbb{R}^{N}$ with $\left|x_{j}\right| \rightarrow \infty$ such that $\varrho \leq\left|z_{j}\left(x_{j}\right)\right|$ for all $j$.

Without loss of generality, we may assume that $z_{j} \rightarrow z$ in $H^{2}$. By (2.7),

$$
\begin{aligned}
\varrho \leq\left|z_{j}\left(x_{j}\right)\right| & \leq C_{0}\left(\int_{B_{1}\left(x_{j}\right)}\left|z_{j}\right|^{2}\right)^{1 / 2} \\
& \leq C_{0}\left(\int_{B_{1}\left(x_{j}\right)}\left|z_{j}-z\right|^{2}\right)^{1 / 2}+C_{0}\left(\int_{B_{1}\left(x_{j}\right)}|z|^{2}\right)^{1 / 2} \rightarrow 0,
\end{aligned}
$$

a contradiction. Therefore, by (2.8) and (2.9), there are $R>0$ and $\delta>0$ such that $\Delta|z|^{2} \geq \delta|z|^{2}$ for $|x| \geq R$. 
Let $\Gamma$ be a fundamental solution to $-\Delta \Gamma+\delta \Gamma=0$. Using the uniform boundedness, we may choose $\Gamma$ so that $|z(y)|^{2} \leq \Gamma(y)$ for $|y|=R$. Set $w=$ $|z|^{2}-\Gamma$, then

$$
\Delta w=\Delta|z|^{2}-\Delta \Gamma \geq \delta\left(|z|^{2}-\Gamma\right)=\delta w .
$$

By the maximum principle, we can conclude that $w(y) \leq 0$ for $|y| \geq R$, i.e.

$$
|z(y)|^{2} \leq \Gamma(y) \text { for }|y| \geq R .
$$

It is well known that there are $c^{\prime}, C^{\prime}>0$ such that

$$
\Gamma(y) \leq C^{\prime} \exp \left(-c^{\prime}|y|\right) \quad \text { for }|y| \geq 1 .
$$

Therefore, there are $c, C>0$ such that

$$
|z(x)|^{2} \leq C \exp (-c|x|) \quad \text { for } x \in \mathbb{R}^{N},
$$

that is,

$$
|z(x)| \leq \sqrt{C} \exp \left(-\frac{c}{2}|x|\right) \quad \text { for } x \in \mathbb{R}^{N} .
$$

As before we introduce the following notations:

$$
\begin{aligned}
& h_{\mu}: E^{+} \rightarrow E^{-}, \quad \Phi_{\mu}\left(z+h_{\mu}(z)\right)=\max _{w \in E^{-}} \Phi_{\mu}(z+w) ; \\
& I_{\mu}: E^{+} \rightarrow \mathbb{R}, \quad I_{\mu}(z)=\Phi_{\mu}\left(z+h_{\mu}(z)\right) ; \\
& \mathscr{N}_{\mu}:=\left\{z \in E^{+} \backslash\{0\}: I_{\mu}^{\prime}(z) z=0\right\} .
\end{aligned}
$$

Plainly, critical points of $I_{\mu}$ and $\Phi_{\mu}$ are in one-to-one correspondence via the injective map $z \mapsto z+h_{\mu}(z)$ from $E^{+}$into $E$. Similar to Lemma 2.8 , it is easy to check that, for each $z \in E^{+} \backslash\{0\}$, there is a unique $t=t(z)>0$ such that $t z \in \mathscr{N}_{\mu}$ and

$$
c_{\mu}=\inf \left\{I_{\mu}(z): z \in \mathscr{N}_{\mu}\right\}=\inf _{e \in E^{+} \backslash\{0\}} \max _{z \in E_{e}} \Phi_{\mu}(z) .
$$

For the later use, define, for $\sigma \in\left(2,2^{*}\right)$,

$$
T_{\sigma}:=\inf _{u \in E^{+} \backslash\{0\}} \max _{v \in E^{-}} \frac{\|u\|^{2}-\|v\|^{2}}{|u+v|_{\sigma}^{2}},
$$

and consider the system

$$
\begin{cases}-\Delta u+b \cdot \nabla u+u=|z|^{\sigma-2} v & \text { in } \mathbb{R}^{N}, \\ -\Delta v-b \cdot \nabla v+v=|z|^{\sigma-2} u & \text { in } \mathbb{R}^{N},\end{cases}
$$

with the energy functional defined by

$$
\mathscr{T}_{\sigma}(z):=\frac{1}{2}\left(\left\|z^{+}\right\|^{2}-\left\|z^{-}\right\|^{2}\right)-\frac{1}{\sigma} \int_{\mathbb{R}^{N}}|z|^{\sigma}
$$

and the least energy denoted by $\gamma$. The following lemma is due to [13, Lemma 4.5] (see also [15]). 
LEMMA 3.2. $T_{\sigma}$ is achieved at some $z$ which is a ground state solution of system (3.3). Moreover,

$$
T_{\sigma}=\left(\frac{2 \sigma \gamma}{\sigma-2}\right)^{(\sigma-2) / \sigma}
$$

Proof. We employ the argument from [13]. For reader's convenience, we give the details. Set, for any $u \in E^{+}$and $v \in E^{-}$,

$$
\mathscr{J}_{u}(v)=\frac{\|u\|^{2}-\|v\|^{2}}{|u+v|_{\sigma}^{2}} \text { and } \quad T_{\sigma}(u)=\max _{v \in E^{-}} \mathscr{J}_{u}(v) .
$$

If $w \in E^{-}$with $\mathscr{J}_{u}(w)=T_{\sigma}(u)$, then for $v \in E^{-}$,

$$
\begin{aligned}
0=\mathscr{J}_{u}^{\prime}(w) v=\frac{2}{|u+w|_{\sigma}^{4}} & \left(-\langle w, v\rangle|u+w|_{\sigma}^{2}\right. \\
& \left.-\left(\|u\|^{2}-\|w\|^{2}\right)|u+w|_{\sigma}^{2-\sigma} \int_{\mathbb{R}^{N}}|u+w|^{\sigma-2}(u+w) v\right)
\end{aligned}
$$

and

$$
\begin{aligned}
\mathscr{J}_{u}^{\prime \prime}(w)[v, v]= & \frac{2}{|u+w|_{\sigma}^{4}}\left(-\|v\|^{2}|u+w|_{\sigma}^{2}\right. \\
& -\left(\|u\|^{2}-\|w\|^{2}\right)|u+w|_{\sigma}^{2-\sigma} \int_{\mathbb{R}^{N}}|u+w|^{\sigma-2}|v|^{2} \\
& +(\sigma-2)\left(\|u\|^{2}-\|w\|^{2}\right)|u+w|_{\sigma}^{2-2 \sigma}\left(\int_{\mathbb{R}^{N}}|u+w|^{\sigma-2}(u+w) v\right)^{2} \\
& \left.-(\sigma-2)\left(\|u\|^{2}-\|w\|^{2}\right)|u+w|_{\sigma}^{2-\sigma} \int_{\mathbb{R}^{N}}|u+w|^{\sigma-4}((u+w) v)^{2}\right) \\
\leq & \frac{2}{|u+w|_{\sigma}^{4}}\left(-\|v\|^{2}|u+w|_{\sigma}^{2} \int_{\mathbb{R}^{N}}|u+w|^{\sigma-2}|v|^{2}\right)<0 .
\end{aligned}
$$

Here we have used the estimate (by the Hölder inequality)

$$
\left(\int_{\mathbb{R}^{N}}|u+w|^{\sigma-2}(u+w) v\right)^{2} \leq|u+w|_{\sigma}^{\sigma} \int_{\mathbb{R}^{N}}|u+w|^{\sigma-4}((u+w) v)^{2}
$$

and $\|u\|^{2}-\|w\|^{2}>0\left(\right.$ since $\left.T_{\sigma}(u)>0\right)$. Therefore, $\mathscr{J}_{u}$ attains its maximum at a unique point. Observe that

$$
T_{\sigma}=\inf _{u \in E^{+} \backslash\{0\}} \max _{w \in E_{u}} \frac{\left\|w^{+}\right\|^{2}-\left\|w^{-}\right\|^{2}}{|w|_{\sigma}^{2}} .
$$

If the function

$$
m_{u}(w)=\frac{\left\|w^{+}\right\|^{2}-\left\|w^{-}\right\|^{2}}{|w|_{\sigma}^{2}}
$$


attains its maximum on $E_{u}$ at $w$, setting $\widehat{w}=m_{u}(w)^{1 /(\sigma-2)} w /|w|_{\sigma}$, then $m_{u}(\widehat{w})$ $=m_{u}(w)$ and for any $v \in E_{u}$,

$$
0=m_{u}^{\prime}(w) v=2\left(\frac{m_{u}(w)^{1 /(\sigma-2)}}{|w|_{\sigma}}\right)^{-1}\left((A \widehat{w}, v)-\int_{\mathbb{R}^{N}}|\widehat{w}|^{\sigma-2} \widehat{w} v\right) .
$$

This implies that $\widehat{w}$ is a critical point of $\mathscr{T}_{\sigma}$. Consequently,

$$
\gamma \leq \mathscr{T}_{\sigma}(\widehat{w})=\frac{\sigma-2}{2 \sigma}\left(m_{u}(\widehat{w})\right)^{\sigma /(\sigma-2)}=\frac{\sigma-2}{2 \sigma}\left(m_{u}(w)\right)^{\sigma /(\sigma-2)},
$$

hence, $T_{\sigma} \geq(2 \sigma \gamma /(\sigma-2))^{(\sigma-2) / \sigma}$.

One the other hand, let $z$ be a ground state solution of system (3.3) with $z=z^{+}+z^{-}$and take $u=z^{+}$. One has $m_{u}(z)=|z|_{\sigma}^{\sigma-2}$. Plainly, one checks as above that for $v \in E^{-}$,

and

$$
\mathscr{J}_{u}^{\prime}\left(z^{-}\right) v=\frac{2}{|z|_{\sigma}^{2}}\left(-\left\langle z^{-}, v\right\rangle-\int_{\mathbb{R}^{N}}|z|^{\sigma-2} z v\right)=0
$$

$\mathscr{J}_{u}^{\prime \prime}\left(z^{-}\right)[v, v] \leq \frac{2}{|z|_{\sigma}^{4}}\left(-\|v\|^{2}|z|_{\sigma}^{2}-\left(\left\|z^{+}\right\|^{2}-\left\|z^{-}\right\|^{2}\right)|z|_{\sigma}^{2-\sigma} \int_{\mathbb{R}^{N}}|z|^{\sigma-2}|v|^{2}\right)<0$.

Therefore,

$$
T_{\sigma}\left(z^{+}\right)=\frac{\left\|z^{+}\right\|^{2}-\left\|z^{-}\right\|^{2}}{|z|_{\sigma}^{2}}=|z|_{\sigma}^{\sigma-2}=\left(\frac{2 \sigma \gamma}{\sigma-2}\right)^{(\sigma-2) / \sigma} .
$$

Lemma 3.3. If $f(s)=c_{0} s^{\sigma-2}$, then the corresponding least energy of system (3.1) denoted by $c_{\mu}(\sigma)$ satisfies $c_{\mu}(\sigma) \leq\left(\mu c_{0}\right)^{-2 /(\sigma-2)} \gamma$.

Proof. Now assume $f(s)=c_{0} s^{\sigma-2}$, and denote the corresponding energy functional of system (3.1) by

$$
\mathscr{T}^{\mu}(z):=\frac{1}{2}\left(\left\|z^{+}\right\|^{2}-\left\|z^{-}\right\|^{2}\right)-\frac{\mu c_{0}}{\sigma} \int_{\mathbb{R}^{N}}|z|^{\sigma} .
$$

Let $z$ be a least energy solution of system (3.3) and $u=z^{+}$. Set $e_{\sigma} \in E_{u}$ with $\mathscr{T}^{\mu}\left(e_{\sigma}\right)=\max _{w \in E_{u}} \mathscr{T}^{\mu}(w)$. Then by Lemma 3.2,

$$
\begin{aligned}
c_{\mu}(\sigma) & \leq \mathscr{T}^{\mu}\left(e_{\sigma}\right)=\frac{\sigma-2}{2 \sigma}\left(\mu c_{0}\right)^{-2 /(\sigma-2)}\left(\frac{\left\|e_{\sigma}^{+}\right\|^{2}-\left\|e_{\sigma}^{-}\right\|^{2}}{\left|e_{\sigma}\right|_{\sigma}^{2}}\right)^{\sigma /(\sigma-2)} \\
& \leq \frac{\sigma-2}{2 \sigma}\left(\mu c_{0}\right)^{-2 /(\sigma-2)} T_{\sigma}^{\sigma /(\sigma-2)}=\left(\mu c_{0}\right)^{-2 /(\sigma-2)} \gamma,
\end{aligned}
$$

completing the proof.

We now turn to critical system (3.2). Its solutions are critical points of the functional

$$
\begin{aligned}
\Phi_{\mu \nu}(z) & =\frac{1}{2}\left(\left\|z^{+}\right\|^{2}-\left\|z^{-}\right\|^{2}\right)-\mu \int_{\mathbb{R}^{N}} F(|z|)-\frac{\nu}{2^{*}} \int_{\mathbb{R}^{N}}|z|^{2^{*}} \\
& :=\frac{1}{2}\left(\left\|z^{+}\right\|^{2}-\left\|z^{-}\right\|^{2}\right)-\Psi_{\mu \nu}(z)
\end{aligned}
$$


defined for $z=z^{-}+z^{+} \in E=E^{-} \oplus E^{+}$. Denote the critical set, the least energy, and the set of ground state solutions of $\Phi_{\mu \nu}$ as follows

$$
\begin{aligned}
\mathscr{K}_{\mu \nu} & :=\left\{z \in E: \Phi_{\mu \nu}^{\prime}(z)=0\right\}, \\
c_{\mu \nu} & :=\inf \left\{\Phi_{\mu \nu}(z): z \in \mathscr{K}_{\mu \nu} \backslash\{0\}\right\}, \\
\mathscr{L}_{\mu \nu} & :=\left\{z \in \mathscr{K}_{\mu \nu}: \Phi_{\mu \nu}(z)=c_{\mu \nu}\right\} .
\end{aligned}
$$

First we have the following result, which is very important in our arguments.

LEMMA 3.4. $c_{\mu \nu}$ is attained if $c_{\mu \nu}\left\langle c^{*}:=S^{N / 2} / N \nu^{(N-2) / 2}\right.$.

Proof. Let $\left\{z_{n}\right\}$ be a (PS) $c$ sequence with $c=c_{\mu \nu}$, then $\Phi_{\mu \nu}\left(z_{n}\right) \rightarrow c$ and $\Phi_{\mu \nu}^{\prime}\left(z_{n}\right) \rightarrow 0$ as $n \rightarrow \infty$. It is not difficult to check that $\left\{z_{n}\right\}$ is bounded in $E$. By Lion's concentration principle [25], $\left\{z_{n}\right\}$ is either vanishing or non-vanishing.

Assume that $\left\{z_{n}\right\}$ is vanishing. Then $\left|z_{n}\right|_{s} \rightarrow 0$ for $s \in\left(2,2^{*}\right)$. By (2.2), we have

$\int_{\mathbb{R}^{N}} F\left(\left|z_{n}\right|\right)=o(1), \quad \int_{\mathbb{R}^{N}} f\left(\left|z_{n}\right|\right)\left|z_{n}\right|^{2}=o(1), \quad \int_{\mathbb{R}^{N}} f\left(\left|z_{n}\right|\right) z_{n}\left(z_{n}^{+}-z_{n}^{-}\right)=o(1)$.

Then

$$
\begin{aligned}
c & +o(1)=\Phi_{\mu \nu}\left(z_{n}\right)-\frac{1}{2} \Phi_{\mu \nu}^{\prime}\left(z_{n}\right) z_{n} \\
& =\mu \int_{\mathbb{R}^{N}}\left(\frac{1}{2} f\left(\left|z_{n}\right|\right)\left|z_{n}\right|^{2}-F\left(\left|z_{n}\right|\right)\right)+\nu\left(\frac{1}{2}-\frac{1}{2^{*}}\right)\left|z_{n}\right|_{2^{*}}^{2^{*}}=\frac{\nu}{N}\left|z_{n}\right|_{2^{*}}^{2^{*}}+o(1)
\end{aligned}
$$

which yields that $\left|z_{n}\right|_{2^{*}} \rightarrow(N c / \nu)^{1 / 2^{*}}$ as $n \rightarrow \infty$. Moreover, by the Hölder inequality, we have

$$
\begin{aligned}
\left\|z_{n}\right\|^{2}= & \Phi_{\mu \nu}^{\prime}\left(z_{n}\right)\left(z_{n}^{+}-z_{n}^{-}\right) \\
& +\mu \int_{\mathbb{R}^{N}} f\left(\left|z_{n}\right|\right) z_{n}\left(z_{n}^{+}-z_{n}^{-}\right)+\nu \int_{\mathbb{R}^{N}}\left|z_{n}\right|^{2^{*}-2} z_{n}\left(z_{n}^{+}-z_{n}^{-}\right) \\
= & \nu \int_{\mathbb{R}^{N}}\left|z_{n}\right|^{2^{*}-2} z_{n}\left(z_{n}^{+}-z_{n}^{-}\right)+o(1) \leq \nu\left|z_{n}\right|_{2^{*}}^{2^{*}-1}\left|z_{n}^{+}-z_{n}^{-}\right|_{2^{*}}+o(1),
\end{aligned}
$$

combining with $\left\|z_{n}\right\|=\left\|z_{n}^{+}-z_{n}^{-}\right\|$, which implies that

$$
\begin{aligned}
S & \leq \frac{\left\|z_{n}\right\|}{\left|z_{n}\right| 2_{2^{*}}} \frac{\left\|z_{n}^{+}-z_{n}^{-}\right\|}{\left|z_{n}^{+}-z_{n}^{-}\right|_{2^{*}}} \leq \nu\left|z_{n}\right|_{2^{*}}^{2^{*}-2}+o(1) \\
& \leq \nu\left(\frac{N c}{\nu}\right)^{\left(2^{*}-2\right) / 2^{*}}+o(1)=\nu^{(N-2) / N}(N c)^{2 / N}+o(1),
\end{aligned}
$$

and hence $c \geq S^{N / 2} / N \nu^{(N-2) / 2}$, a contradiction.

Therefore, $\left\{z_{n}\right\}$ is non-vanishing, that is, there exist $r, \vartheta>0$ and $x_{n} \in \mathbb{R}^{N}$ such that, setting $w_{n}(x)=z_{n}\left(x+x_{n}\right)$, along a subsequence,

$$
\int_{B_{r}(0)}\left|w_{n}\right|^{2} \geq \vartheta
$$


Without loss of generality, we assume $w_{n} \rightarrow w$. Then $w \neq 0$ and is a solution of system (3.2). And so $c_{\mu \nu}$ is attained.

LEMMA 3.5. $c_{\mu \nu}$ is attained if

$$
\mu^{-1} \nu^{(N-2)(\sigma-2) / 4}<\mathscr{R}_{\sigma} .
$$

Proof. Observe that, for the nonlinearities, by $\left(\mathrm{F}_{2}\right)$ we have

$$
\Psi_{\mu \nu}(z) \geq \Psi_{\mu}(z) \geq \frac{\mu c_{0}}{\sigma} \int_{\mathbb{R}^{N}}|z|^{\sigma} .
$$

So, by the reduction process and the min-max scheme, we deduce $c_{\mu \nu} \leq c_{\mu} \leq$ $c_{\mu}(\sigma)$. If $\left(\mu c_{0}\right)^{-2 /(\sigma-2)} \gamma<c^{*}$, that is, (3.4) is satisfied, then $c_{\mu \nu}<c^{*}$ by Lemma 3.3. So $c_{\mu \nu}$ is attained by Lemma 3.4.

Combining Lemma 3.5, similar to the proof of Lemma 3.1, we have the following results.

LEMMA 3.6. If (3.4) is satisfied, then:

(a) $\mathscr{K}_{\mu \nu} \neq \emptyset$;

(b) $c_{\mu \nu}>0$ is attained and $\mathscr{L}_{\mu \nu}$ is compact in $H^{2}\left(\mathbb{R}^{N}\right)$;

(c) there exist $C, c>0$ such that $|z(x)| \leq C \exp (-c|x|)$ for all $x \in \mathbb{R}^{N}$ and $z \in \mathscr{L}_{\mu \nu}$.

Set as before the induced map $h_{\mu \nu}: E^{+} \rightarrow E^{-}$, the functional

$$
I_{\mu \nu} \in C^{1}\left(E^{+}, \mathbb{R}\right): I_{\mu \nu}(z)=\Phi_{\mu \nu}\left(z+h_{\mu \nu}(z)\right),
$$

and the Nehari manifold $\mathscr{N}_{\mu \nu}$. The following lemmas will be useful to study our problem.

Lemma 3.7. Let $z \in \mathscr{N}_{\mu \nu}$ be such that $I_{\mu \nu}(z)=c_{\mu \nu}$ and set $E_{z}=E^{-} \oplus \mathbb{R} z$. Then

$$
\max _{w \in E_{z}} \Phi_{\mu \nu}(w)=I_{\mu \nu}(z) .
$$

Proof. Clearly, since $z+h_{\mu \nu}(z) \in E_{z}$,

$$
I_{\mu \nu}(z)=\Phi_{\mu \nu}\left(z+h_{\mu \nu}(z)\right) \leq \max _{w \in E_{z}} \Phi_{\mu \nu}(w) .
$$

On the other hand, for any $w=v+s z \in E_{z}$,

$$
\Phi_{\mu \nu}(w)=\Phi_{\mu \nu}(v+s z) \leq \Phi_{\mu \nu}\left(s z+h_{\mu \nu}(s z)\right)=I_{\mu \nu}(s z) .
$$

Thus, since $z \in \mathscr{N}_{\mu \nu}$,

$$
\max _{w \in E_{z}} \Phi_{\mu \nu}(w) \leq \max _{s \geq 0} I_{\mu \nu}(s z)=I_{\mu \nu}(z) .
$$

LEMMA 3.8. Let $\mu_{1} \leq \mu_{2}$ and $\nu_{1} \leq \nu_{2}$, then $c_{\mu_{1} \nu_{1}} \geq c_{\mu_{2} \nu_{2}}$. In addition, if $\max \left\{\mu_{2}-\mu_{1}, \nu_{2}-\nu_{1}\right\}>0$, then $c_{\mu_{1} \nu_{1}}>c_{\mu_{2} \nu_{2}}$. 
PROOF. Let $z \in \mathscr{L}_{\mu_{1} \nu_{1}}$ with $\Phi_{\mu_{1} \nu_{1}}(z)=c_{\mu_{1} \nu_{1}}$ and set $e=z^{+}$. Then

$$
c_{\mu_{1} \nu_{1}}=\Phi_{\mu_{1} \nu_{1}}(z)=\max _{w \in E_{e}} \Phi_{\mu_{1} \nu_{1}}(w) .
$$

Let $z_{0} \in E_{e}$ be such that $\Phi_{\mu_{2} \nu_{2}}\left(z_{0}\right)=\max _{w \in E_{e}} \Phi_{\mu_{2} \nu_{2}}(w)$. One has

$$
\begin{aligned}
c_{\mu_{1} \nu_{1}} & =\Phi_{\mu_{1} \nu_{1}}(z) \geq \Phi_{\mu_{1} \nu_{1}}\left(z_{0}\right) \\
& =\Phi_{\mu_{2} \nu_{2}}\left(z_{0}\right)+\left(\mu_{2}-\mu_{1}\right) \int_{\mathbb{R}^{N}} F\left(\left|z_{0}\right|\right)+\frac{\nu_{2}-\nu_{1}}{2^{*}} \int_{\mathbb{R}^{N}}\left|z_{0}\right|^{2^{*}} \\
& \geq c_{\mu_{2} \nu_{2}}+\left(\mu_{2}-\mu_{1}\right) \int_{\mathbb{R}^{N}} F\left(\left|z_{0}\right|\right)+\frac{\nu_{2}-\nu_{1}}{2^{*}} \int_{\mathbb{R}^{N}}\left|z_{0}\right|^{2^{*}},
\end{aligned}
$$

this implies $c_{\mu_{1} \nu_{1}} \geq c_{\mu_{2} \nu_{2}}$.

\section{Proof of the main result}

In the sequel, we shall denote $\mu=K(0)$ and $\nu=W(0)$, remark that by $\left(\mathrm{K}_{0}\right)$

$$
K(\varepsilon x) \rightarrow \mu \quad \text { and } \quad W(\varepsilon x) \rightarrow \nu
$$

uniformly on bounded sets of $x$ as $\varepsilon \rightarrow 0$. Recall that

$$
\kappa:=\max _{x \in \mathbb{R}^{N}} K(x), \quad \omega:=\max _{x \in \mathbb{R}^{N}} W(x),
$$

and let

$$
\Phi_{\kappa \omega}(z)=\frac{1}{2}\left(\left\|z^{+}\right\|^{2}-\left\|z^{-}\right\|^{2}\right)-\kappa \int_{\mathbb{R}^{N}} F(|z|)-\frac{\omega}{2^{*}} \int_{\mathbb{R}^{N}}|z|^{2^{*}} .
$$

The following lemma plays an important role, which is the key ingredient for existence result.

LEMMA 4.1. $\limsup _{\varepsilon \rightarrow 0} c_{\varepsilon} \leq c_{\mu \nu}$. In particular, $\lim _{\varepsilon \rightarrow 0} c_{\varepsilon}=c_{\kappa \omega}$ if $\mathscr{M} \neq \emptyset$.

Proof. Firstly we show that

$$
c_{\varepsilon} \geq c_{\kappa \omega} \text { for all } \varepsilon>0 .
$$

Arguing indirectly, assume that $c_{\varepsilon}<c_{\kappa \omega}$ for some $\varepsilon>0$. By definition and Lemma 2.9, we can choose $e \in E^{+} \backslash\{0\}$ such that $\max _{z \in E_{e}} \Phi_{\varepsilon}(z)<c_{\kappa \omega}$. By definition again one has $c_{\kappa \omega} \leq \max _{z \in E_{e}} \Phi_{\kappa \omega}(z)$. Since $K_{\varepsilon}(x) \leq \kappa$ and $W_{\varepsilon}(x) \leq \omega$, then $\Phi_{\varepsilon}(z) \geq \Phi_{\kappa \omega}(z)$ for all $z \in E$, and we get

$$
c_{\kappa \omega}>\max _{z \in E_{e}} \Phi_{\varepsilon}(z) \geq \max _{z \in E_{e}} \Phi_{\kappa \omega}(z) \geq c_{\kappa \omega},
$$

which is a contradiction.

Now we turn to the proof of the desired conclusion. Set $K^{0}(x)=\mu-K(x)$, $W^{0}(x)=\nu-W(x)$ and $K_{\varepsilon}^{0}(x)=K^{0}(\varepsilon x), W_{\varepsilon}^{0}(x)=W^{0}(\varepsilon x)$. Then

$$
\Phi_{\varepsilon}(z)=\Phi_{\mu \nu}(z)+\int_{\mathbb{R}^{N}} K_{\varepsilon}^{0}(x) F(|z|)+\frac{1}{2^{*}} \int_{\mathbb{R}^{N}} W_{\varepsilon}^{0}(x)|z|^{2^{*}} .
$$


In virtue of Lemma 3.6, let $z \in \mathscr{L}_{\mu \nu}$ be a ground state solution to (3.2) and $e=z^{+}$. Obviously, $e \in \mathscr{N}_{\mu \nu}, z^{-}=h_{\mu \nu}(e)$ and $I_{\mu \nu}(e)=c_{\mu \nu}$. There is a unique $t_{\varepsilon}$ such that $t_{\varepsilon} e \in \mathscr{N}_{\varepsilon}$, and hence

$$
c_{\varepsilon} \leq I_{\varepsilon}\left(t_{\varepsilon} e\right) .
$$

By Lemma 2.10, $\left\{t_{\varepsilon}\right\}$ is bounded. Without loss of generality, we may assume that $t_{\varepsilon} \rightarrow t_{0}$ as $\varepsilon \rightarrow 0$. Set

$$
\begin{gathered}
z_{\varepsilon}=t_{\varepsilon} e+h_{\varepsilon}\left(t_{\varepsilon} e\right), \quad w_{\varepsilon}=t_{\varepsilon} e+h_{\mu \nu}\left(t_{\varepsilon} e\right), \\
v_{\varepsilon}=w_{\varepsilon}-z_{\varepsilon}=h_{\mu \nu}\left(t_{\varepsilon} e\right)-h_{\varepsilon}\left(t_{\varepsilon} e\right) .
\end{gathered}
$$

Next, we show that $v_{\varepsilon} \rightarrow 0$ in $E$. Similarly to (2.4), we have

$$
\begin{aligned}
\Phi_{\varepsilon}\left(z_{\varepsilon}\right)-\Phi_{\varepsilon}\left(w_{\varepsilon}\right) & =\frac{1}{2}\left\|v_{\varepsilon}\right\|^{2}+\int_{\mathbb{R}^{N}} H_{\varepsilon}(x) \geq \frac{1}{2}\left\|v_{\varepsilon}\right\|^{2}, \\
\Phi_{\mu \nu}\left(w_{\varepsilon}\right)-\Phi_{\mu \nu}\left(z_{\varepsilon}\right) & =\frac{1}{2}\left\|v_{\varepsilon}\right\|^{2}+\int_{\mathbb{R}^{N}} G_{\varepsilon}(x) \geq \frac{1}{2}\left\|v_{\varepsilon}\right\|^{2},
\end{aligned}
$$

where

$$
\begin{aligned}
H_{\varepsilon}(x)= & \int_{0}^{1}(1-t)\left(K _ { \varepsilon } ( x ) \left[f^{\prime}\left(\left|z_{\varepsilon}+t v_{\varepsilon}\right|\right) \frac{\left|\left(z_{\varepsilon}+t v_{\varepsilon}\right) v_{\varepsilon}\right|^{2}}{\left|z_{\varepsilon}+t v_{\varepsilon}\right|}\right.\right. \\
& \left.\left.+f\left(\left|z_{\varepsilon}+t v_{\varepsilon}\right|\right)\left|v_{\varepsilon}\right|^{2}\right]+\left(2^{*}-1\right) W_{\varepsilon}(x)\left|z_{\varepsilon}+t v_{\varepsilon}\right|^{2^{*}-2}\left|v_{\varepsilon}\right|^{2}\right) d t, \\
G_{\varepsilon}(x)= & \int_{0}^{1}(1-t)\left(\mu \left[f^{\prime}\left(\left|w_{\varepsilon}-t v_{\varepsilon}\right|\right) \frac{\left|\left(w_{\varepsilon}-t v_{\varepsilon}\right) v_{\varepsilon}\right|^{2}}{\left|w_{\varepsilon}-t v_{\varepsilon}\right|}\right.\right. \\
& \left.\left.+f\left(\left|w_{\varepsilon}-t v_{\varepsilon}\right|\right)\left|v_{\varepsilon}\right|^{2}\right]+\left(2^{*}-1\right) \nu\left|w_{\varepsilon}-t v_{\varepsilon}\right|^{2^{*}-2}\left|v_{\varepsilon}\right|^{2}\right) d t .
\end{aligned}
$$

From (4.5), (4.6) and the mean value theorem we know that

$$
\begin{aligned}
\left\|v_{\varepsilon}\right\|^{2} \leq \Phi_{\varepsilon}\left(z_{\varepsilon}\right)-\Phi_{\varepsilon}\left(w_{\varepsilon}\right)+\Phi_{\mu \nu}\left(w_{\varepsilon}\right)-\Phi_{\mu \nu}\left(z_{\varepsilon}\right) \\
=\int_{\mathbb{R}^{N}} K_{\varepsilon}^{0}(x)\left(F\left(\left|z_{\varepsilon}\right|\right)-F\left(\left|w_{\varepsilon}\right|\right)\right)+\frac{1}{2^{*}} \int_{\mathbb{R}^{N}} W_{\varepsilon}^{0}(x)\left(\left|z_{\varepsilon}\right|^{2^{*}}-\left|w_{\varepsilon}\right|^{2^{*}}\right) \\
=-\int_{\mathbb{R}^{N}} K_{\varepsilon}^{0}(x) f\left(\left|w_{\varepsilon}\right|\right) w_{\varepsilon} v_{\varepsilon}-\int_{\mathbb{R}^{N}} W_{\varepsilon}^{0}(x)\left|w_{\varepsilon}\right|^{2^{*}-2} w_{\varepsilon} v_{\varepsilon} \\
\quad+\int_{\mathbb{R}^{N}} K_{\varepsilon}^{0}(x) \int_{0}^{1}(1-t)\left(\left[f^{\prime}\left(\left|w_{\varepsilon}-t v_{\varepsilon}\right|\right) \frac{\left|\left(w_{\varepsilon}-t v_{\varepsilon}\right) v_{\varepsilon}\right|^{2}}{\left|w_{\varepsilon}-t v_{\varepsilon}\right|}+f\left(\left|w_{\varepsilon}-t v_{\varepsilon}\right|\right)\left|v_{\varepsilon}\right|^{2}\right]\right) d t \\
\quad+\int_{\mathbb{R}^{N}} W_{\varepsilon}^{0}(x) \int_{0}^{1}(1-t)\left(\left(2^{*}-1\right)\left|w_{\varepsilon}-t v_{\varepsilon}\right|^{2^{*}-2}\left|v_{\varepsilon}\right|^{2}\right) d t \\
\leq-\int_{\mathbb{R}^{N}} K_{\varepsilon}^{0}(x) f\left(\left|w_{\varepsilon}\right|\right) w_{\varepsilon} v_{\varepsilon}-\int_{\mathbb{R}^{N}} W_{\varepsilon}^{0}(x)\left|w_{\varepsilon}\right|^{2^{*}-2} w_{\varepsilon} v_{\varepsilon} .
\end{aligned}
$$


It follows from $\left(\mathrm{F}_{1}\right)$ that

$$
\begin{aligned}
\left\|v_{\varepsilon}\right\|^{2} \leq & \int_{\mathbb{R}^{N}}\left|K_{\varepsilon}^{0}(x)\right|\left|f\left(\left|w_{\varepsilon}\right|\right)\right|\left|w_{\varepsilon} v_{\varepsilon}\right|+\int_{\mathbb{R}^{N}}\left|W_{\varepsilon}^{0}(x)\right|\left|w_{\varepsilon}\right|^{2^{*}-2}\left|w_{\varepsilon} v_{\varepsilon}\right| \\
\leq & c_{1} \int_{\mathbb{R}^{N}}\left|K_{\varepsilon}^{0}(x)\right|\left|w_{\varepsilon}\right|\left|v_{\varepsilon}\right| \\
& +c_{1} \int_{\mathbb{R}^{N}}\left|K_{\varepsilon}^{0}(x)\right|\left|w_{\varepsilon}\right|^{p-1}\left|v_{\varepsilon}\right|+\int_{\mathbb{R}^{N}}\left|W_{\varepsilon}^{0}(x)\right|\left|w_{\varepsilon}\right|^{2^{*}-1}\left|v_{\varepsilon}\right| \\
\leq & c_{2}\left(\int_{\mathbb{R}^{N}}\left|K_{\varepsilon}^{0}(x) w_{\varepsilon}\right|^{2}\right)^{1 / 2}\left|v_{\varepsilon}\right|_{2} \\
& +c_{1}\left(\int_{\mathbb{R}^{N}}\left|K_{\varepsilon}^{0}(x)\right|^{p /(p-1)}\left|w_{\varepsilon}\right|^{p}\right)^{(p-1) / p}\left|v_{\varepsilon}\right|_{p} \\
& +\left(\int_{\mathbb{R}^{N}}\left|W_{\varepsilon}^{0}(x)\right|^{2^{*} /\left(2^{*}-1\right)}\left|w_{\varepsilon}\right|^{2^{*}}\right)^{\left(2^{*}-1\right) / 2^{*}}\left|v_{\varepsilon}\right|_{2^{*} .}
\end{aligned}
$$

Since $t_{\varepsilon} \rightarrow t_{0}$, it is clear that $\left\{z_{\varepsilon}\right\},\left\{w_{\varepsilon}\right\}$ and $\left\{v_{\varepsilon}\right\}$ are bounded. Moreover, by the exponential decay of $e$ and the continuity of $h_{\mu \nu}$, we have for $q \in\left[2,2^{*}\right]$

$$
\limsup _{R \rightarrow \infty} \int_{|x|>R}\left|w_{\varepsilon}\right|^{q}=\limsup _{R \rightarrow \infty} \int_{|x|>R}\left|t_{\varepsilon} e+h_{\mu \nu}\left(t_{\varepsilon} e\right)\right|^{q}=0 .
$$

By (4.1) and the above fact, it follows that

$$
\begin{aligned}
\int_{\mathbb{R}^{N}}\left|K_{\varepsilon}^{0}(x)\right|^{2}\left|w_{\varepsilon}\right|^{2} & =\left(\int_{|x| \leq R}+\int_{|x|>R}\right)\left|K_{\varepsilon}^{0}(x)\right|^{2}\left|w_{\varepsilon}\right|^{2} \\
& =\int_{|x| \leq R}\left|K_{\varepsilon}^{0}(x)\right|^{2}\left|w_{\varepsilon}\right|^{2}+c_{3} \int_{|x|>R}\left|w_{\varepsilon}\right|^{2}=o(1)
\end{aligned}
$$

as $\varepsilon \rightarrow 0$, and similarly

$$
\int_{\mathbb{R}^{N}}\left|K_{\varepsilon}^{0}(x)\right|^{p /(p-1)}\left|w_{\varepsilon}\right|^{p}=o(1), \quad \int_{\mathbb{R}^{N}}\left|W_{\varepsilon}^{0}(x)\right|^{2^{*} /\left(2^{*}-1\right)}\left|w_{\varepsilon}\right|^{2^{*}}=o(1)
$$

as $\varepsilon \rightarrow 0$. Thus, by (4.7), (4.8) and (4.9), we know that $\left\|v_{\varepsilon}\right\|=\| h_{\mu \nu}\left(t_{\varepsilon} e\right)-$ $h_{\varepsilon}\left(t_{\varepsilon} e\right) \| \rightarrow 0$, that is, $h_{\varepsilon}\left(t_{\varepsilon} e\right) \rightarrow h_{\mu \nu}\left(t_{0} e\right)$. Consequently,

$$
\int_{\mathbb{R}^{N}} K_{\varepsilon}^{0}(x) F\left(\left|z_{\varepsilon}\right|\right)+\frac{1}{2^{*}} \int_{\mathbb{R}^{N}} W_{\varepsilon}^{0}(x)\left|z_{\varepsilon}\right|^{2^{*}} \rightarrow 0
$$

as $\varepsilon \rightarrow 0$. This, jointly with (4.3), implies

$\Phi_{\varepsilon}\left(z_{\varepsilon}\right)=\Phi_{\varepsilon}\left(t_{\varepsilon} e+h_{\varepsilon}\left(t_{\varepsilon} e\right)\right)=\Phi_{\mu \nu}\left(t_{\varepsilon} e+h_{\varepsilon}\left(t_{\varepsilon} e\right)\right)+o(1)=\Phi_{\mu \nu}\left(t_{0} e+h_{\mu \nu}\left(t_{0} e\right)\right)+o(1)$,

that is, $I_{\varepsilon}\left(t_{\varepsilon} e\right)=I_{\mu \nu}\left(t_{0} e\right)+o(1)$ as $\varepsilon \rightarrow 0$. Recall that by Lemma 3.7

$$
I_{\mu \nu}\left(t_{0} e\right) \leq \max _{w \in E_{e}} \Phi_{\mu \nu}(w)=I_{\mu \nu}(e)=c_{\mu \nu}
$$

Now, using (4.4) we obtain

$$
\lim _{\varepsilon \rightarrow 0} c_{\varepsilon} \leq \lim _{\varepsilon \rightarrow 0} I_{\varepsilon}\left(t_{\varepsilon} e\right)=I_{\mu \nu}\left(t_{0} e\right) \leq c_{\mu \nu}
$$


Suppose additionally $\mathscr{M} \neq \emptyset$ (that is $0 \in \mathscr{M}$ ), we find $\mu=\kappa$ and $\nu=\omega$, then $c_{\mu \nu}=c_{\kappa \omega}$. Obviously, by (4.2) we have $\lim _{\varepsilon \rightarrow 0} c_{\varepsilon}=c_{\kappa \omega}$.

Lemma 4.2. $c_{\varepsilon}$ is attained for all small $\varepsilon>0$.

Proof. Given $\varepsilon>0$, let $\left\{z_{n}\right\} \subset \mathscr{N}_{\varepsilon}$ be a minimizing sequence $I_{\varepsilon}\left(z_{n}\right) \rightarrow c_{\varepsilon}$. By the Ekeland variational principle, we can assume that $\left\{z_{n}\right\}$ is, in addition, a $(\mathrm{PS})_{c_{\varepsilon}}$ sequence for $I_{\varepsilon}$ on $\mathscr{N}_{\varepsilon}$. A standard argument shows that $\left\{z_{n}\right\}$ is in fact a $(\mathrm{PS})_{c_{\varepsilon}}$ sequence for $I_{\varepsilon}$ on $E^{+}$(see [30]) and $\left\{z_{n}\right\}$ is bounded. Hence $\left\{z_{n}+h_{\varepsilon}\left(z_{n}\right)\right\}$ is a bounded (PS) $c_{\varepsilon}$ sequence for $\Phi_{\varepsilon}$ on $E$. Then we can assume, without loss of generality, that $z_{n}+h_{\varepsilon}\left(z_{n}\right):=w_{n} \rightarrow w_{\varepsilon}=w_{\varepsilon}^{+}+w_{\varepsilon}^{-}$in $E$ with $\Phi_{\varepsilon}^{\prime}\left(w_{\varepsilon}\right)=0$. So we are going to show that $w_{\varepsilon} \neq 0$ for all small $\varepsilon>0$.

For this end, we take $\ell>0$ satisfying $\kappa_{\infty}:=\limsup _{|x| \rightarrow \infty} K(x)<\ell<\kappa:=$ $\max _{x \in \mathbb{R}^{N}} K(x)$, and define $K^{\ell}(x)=\min \{\ell, K(x)\}$. Note that, by $\left(K_{1}\right)$ we refer

$$
\ell^{-1} \omega^{(N-2)(\sigma-2) / 4}<\mathscr{R}_{\sigma} .
$$

And invoking Lemma 3.6, (4.10) implies that $c_{\ell \omega}$ is attained.

Now we consider the functional

$$
\Phi_{\varepsilon}^{\ell}(z)=\frac{1}{2}\left(\left\|z^{+}\right\|^{2}-\left\|z^{-}\right\|^{2}\right)-\int_{\mathbb{R}^{N}} K_{\varepsilon}^{\ell}(x) F(|z|)-\frac{1}{2^{*}} \int_{\mathbb{R}^{N}} W_{\varepsilon}(x)|z|^{2^{*}}
$$

and as before define correspondingly $h_{\varepsilon}^{\ell}: E^{+} \rightarrow E^{-}, I_{\varepsilon}^{\ell}: E^{+} \rightarrow \mathbb{R}, \mathscr{N}_{\varepsilon}^{\ell}, c_{\varepsilon}^{\ell}$ and so on. Following the proof of Lemma 4.1, one finds

$$
\lim _{\varepsilon \rightarrow 0} c_{\varepsilon}^{\ell}=c_{\ell \omega}
$$

Assume by contradiction that there is a sequence $\varepsilon_{j} \rightarrow 0$ with $w_{\varepsilon_{j}}=0$. Then $w_{n}=z_{n}+h_{\varepsilon_{j}}\left(z_{n}\right) \rightarrow 0$ in $E, z_{n} \rightarrow 0$ in $L_{\mathrm{loc}}^{q}$ for $q \in\left[2,2^{*}\right)$, and $w_{n}(x) \rightarrow 0$ almost everywhere on $\mathbb{R}^{N}$. Let $t_{n}>0$ be such that $t_{n} z_{n} \in \mathscr{N}_{\varepsilon_{j}}^{\ell}$. We see that $\left\{t_{n}\right\}$ is bounded and one may assume $t_{n} \rightarrow t_{0}$ as $n \rightarrow \infty$. By assumption $\left(\mathrm{K}_{0}\right)$, the set $A_{\varepsilon_{j}}:=\left\{x \in \mathbb{R}^{N}: K_{\varepsilon_{j}}(x)>\ell\right\}$ is bounded. Additionally, by virtue of Lemma 3.7, one has

$$
\Phi_{\varepsilon_{j}}\left(t_{n} z_{n}+h_{\varepsilon_{j}}^{\ell}\left(t_{n} z_{n}\right)\right) \leq \Phi_{\varepsilon_{j}}\left(t_{n} z_{n}+h_{\varepsilon_{j}}\left(t_{n} z_{n}\right)\right)=I_{\varepsilon_{j}}\left(t_{n} z_{n}\right) \leq I_{\varepsilon_{j}}\left(z_{n}\right) .
$$

Then, we obtain

$$
\begin{aligned}
c_{\varepsilon_{j}}^{\ell} & \leq I_{\varepsilon_{j}}^{\ell}\left(t_{n} z_{n}\right)=\Phi_{\varepsilon_{j}}^{\ell}\left(t_{n} z_{n}+h_{\varepsilon_{j}}^{\ell}\left(t_{n} z_{n}\right)\right) \\
& =\Phi_{\varepsilon_{j}}\left(t_{n} z_{n}+h_{\varepsilon_{j}}^{\ell}\left(t_{n} z_{n}\right)\right)+\int_{\mathbb{R}^{N}}\left(K_{\varepsilon_{j}}(x)-K_{\varepsilon_{j}}^{\ell}(x)\right) F\left(\left|t_{n} z_{n}+h_{\varepsilon_{j}}^{\ell}\left(t_{n} z_{n}\right)\right|\right) \\
& \leq I_{\varepsilon_{j}}\left(z_{n}\right)+\int_{A_{\varepsilon_{j}}}\left(K_{\varepsilon_{j}}(x)-\ell\right) F\left(\left|t_{n} z_{n}+h_{\varepsilon_{j}}^{\ell}\left(t_{n} z_{n}\right)\right|\right)=c_{\varepsilon_{j}}+o(1)
\end{aligned}
$$

as $n \rightarrow \infty$, hence $c_{\varepsilon_{j}}^{\ell} \leq c_{\varepsilon_{j}}$. By (4.11), letting $j \rightarrow \infty$, yields $c_{\ell \omega} \leq c_{\kappa \omega}$ which contradicts with $c_{\kappa \omega}<c_{\ell \omega}$ since $\ell<\kappa$ (see Lemma 3.8). 
In the same way, we obtain

Lemma 4.3. $\mathscr{L}_{\varepsilon}$ is compact in $H^{2}\left(\mathbb{R}^{N}\right)$ for all small $\varepsilon>0$.

Proof. We first show that $\mathscr{L}_{\varepsilon}$ is compact in $E$. Assume by contradiction that, for some $\varepsilon_{j} \rightarrow 0, \mathscr{L}_{\varepsilon_{j}}$ is not compact in $E$. Thus, for each $j$, there exists a sequence $\left\{z_{n}^{j}\right\} \subset \mathscr{L}_{\varepsilon_{j}}$ so that it has no convergent subsequence. But $\left\{z_{n}^{j}\right\}$ is bounded. So, we may assume, without loss of generality, that $z_{n}^{j} \rightarrow 0$ as $n \rightarrow \infty$. As done for proving the Lemma 4.2, one gets a contradiction.

Let $\left\{z_{n}\right\} \subset \mathscr{L}_{\varepsilon}$ such that $z_{n} \rightarrow z$ in $E$. Using the same notations as in (1.6), we have

$$
\begin{aligned}
A z_{n} & =K_{\varepsilon}(x) f\left(\left|z_{n}\right|\right) z_{n}+W_{\varepsilon}(x)\left|z_{n}\right|^{2^{*}-2} z_{n}, \\
A z & =K_{\varepsilon}(x) f(|z|) z+W_{\varepsilon}(x)|z|^{2^{*}-2} z .
\end{aligned}
$$

Therefore, by (2.6) and $z_{n} \rightarrow z$ in $E$, we have

$$
\begin{aligned}
\left|A\left(z_{n}-z\right)\right|_{2}^{2}= & \int_{\mathbb{R}^{N}}\left|K_{\varepsilon}(x)\left(f\left(\left|z_{n}\right|\right) z_{n}-f(|z|) z\right)+W_{\varepsilon}\left(\left|z_{n}\right|^{2^{*}-2} z_{n}-|z|^{2^{*}-2} z\right)\right|^{2} \\
\leq & \int_{\mathbb{R}^{N}}\left|K_{\varepsilon}(x)\left(f\left(\left|z_{n}\right|\right) z_{n}-f(|z|) z\right)\right|^{2} \\
& +\int_{\mathbb{R}^{N}}\left|W_{\varepsilon}\left(\left|z_{n}\right|^{2^{*}-2} z_{n}-|z|^{2^{*}-2} z\right)\right|^{2}=o(1),
\end{aligned}
$$

which implies that $z_{n} \rightarrow z$ in $H^{2}\left(\mathbb{R}^{N}\right)$.

Next we shall prove the concentration phenomena for the ground state solutions of $\left(\mathcal{P}_{\varepsilon}\right)$.

LEMMA 4.4. Let $z_{\varepsilon}$ be given as in the proof of Lemma 4.2, Then there is a maximum point $y_{\varepsilon}$ of $\left|z_{\varepsilon}(x)\right|$ such that $\lim _{\varepsilon \rightarrow 0} \operatorname{dist}\left(\varepsilon y_{\varepsilon}, \mathscr{M}\right)=0$, and for any sequence $y_{\varepsilon}, w_{\varepsilon}(x):=z_{\varepsilon}\left(x+y_{\varepsilon}\right)$ converges in $H^{2}\left(\mathbb{R}^{N}\right)$ to a ground state solution of

$$
\begin{cases}-\Delta u+b \cdot \nabla u+u=\kappa f(|z|) v+\omega|z|^{2^{*}-2} v & \text { in } \mathbb{R}^{N} \\ -\Delta v-b \cdot \nabla v+v=\kappa f(|z|) u+\omega|z|^{2^{*}-2} u & \text { in } \mathbb{R}^{N} .\end{cases}
$$

Proof. Let $\varepsilon_{j} \rightarrow 0, z_{j} \in \mathscr{L}_{j}$, where $\mathscr{L}_{j}=\mathscr{L}_{\varepsilon_{j}}$. Then $\left\{z_{j}\right\}$ is bounded. A concentration argument shows that there exist a sequence $\left\{y_{j}\right\} \subset \mathbb{R}^{N}$ and constants $r>0, \vartheta>0$ such that

$$
\liminf _{j \rightarrow \infty} \int_{B_{r}\left(y_{j}\right)}\left|z_{j}\right|^{2} \geq \vartheta
$$

Set $w_{j}(x)=z_{j}\left(x+y_{j}\right)$. Then $w_{j}=\left(u_{j}, v_{j}\right)$ is a ground state solution to the following system:

$$
\begin{cases}-\Delta u_{j}+b \cdot \nabla u_{j}+u_{j}=\widehat{K}_{\varepsilon_{j}}(x) f\left(\left|w_{j}\right|\right) v_{j}+\widehat{W}_{\varepsilon_{j}}(x)\left|w_{j}\right|^{2^{*}-2} v_{j} & \text { in } \mathbb{R}^{N} \\ -\Delta v_{j}-b \cdot \nabla v_{j}+v_{j}=\widehat{K}_{\varepsilon_{j}}(x) f\left(\left|w_{j}\right|\right) u_{j}+\widehat{W}_{\varepsilon_{j}}(x)\left|w_{j}\right|^{2^{*}-2} u_{j} & \text { in } \mathbb{R}^{N}\end{cases}
$$


where $\widehat{K}_{\varepsilon_{j}}(x)=K\left(\varepsilon_{j}\left(x+y_{j}\right)\right)$ and $\widehat{W}_{\varepsilon_{j}}(x)=W\left(\varepsilon_{j}\left(x+y_{j}\right)\right)$. Hence, for any $\varphi \in E$,

$$
\begin{aligned}
\widehat{\Phi}_{\varepsilon_{j}}^{\prime}\left(w_{j}\right) \varphi= & \left(w_{j}^{+}, \varphi^{+}\right)-\left(w_{j}^{-}, \varphi^{-}\right) \\
& -\int_{\mathbb{R}^{N}} \widehat{K}_{\varepsilon_{j}}(x) f\left(\left|w_{j}\right|\right) w_{j} \varphi-\int_{\mathbb{R}^{N}} \widehat{W}_{\varepsilon_{j}}(x)\left|w_{j}\right|^{2^{*}-2} w_{j} \varphi=0
\end{aligned}
$$

and the least energy (using the notations of the previous section)

$$
\begin{aligned}
\widehat{c}_{\varepsilon_{j}} & =\widehat{\Phi}_{\varepsilon_{j}}\left(w_{j}\right) \\
& =\frac{1}{2}\left(\left\|w_{j}^{+}\right\|^{2}-\left\|w_{j}^{-}\right\|^{2}\right)-\int_{\mathbb{R}^{N}} \widehat{K}_{\varepsilon_{j}}(x) F\left(\left|w_{j}\right|\right)-\frac{1}{2^{*}} \int_{\mathbb{R}^{N}} \widehat{W}_{\varepsilon_{j}}(x)\left|w_{j}\right|^{2^{*}} \\
& =\widehat{\Phi}_{\varepsilon_{j}}\left(w_{j}\right)-\frac{1}{2} \widehat{\Phi}_{\varepsilon_{j}}^{\prime}\left(w_{j}\right) w_{j} \\
& =\int_{\mathbb{R}^{N}} \widehat{K}_{\varepsilon_{j}}(x) \widetilde{F}\left(\left|w_{j}\right|\right)+\frac{1}{N} \int_{\mathbb{R}^{N}} \widehat{W}_{\varepsilon_{j}}(x)\left|w_{j}\right|^{2^{*}}
\end{aligned}
$$

where $\widetilde{F}(|z|)=f(|z|)|z|^{2} / 2-F(|z|)$. Moreover, $\widehat{c}_{\varepsilon_{j}}=\widehat{\Phi}_{\varepsilon_{j}}\left(w_{j}\right)=\Phi_{\varepsilon_{j}}\left(z_{j}\right)=c_{\varepsilon_{j}}$. After extracting a subsequence, we may assume that $w_{j} \rightarrow w$ in $E$ and $w_{j} \rightarrow w$ in $L_{\text {loc }}^{q}$ for $q \in\left[2,2^{*}\right)$ and $w_{j}(x) \rightarrow w(x)$ almost everywhere on $\mathbb{R}^{N}$.

We now turn to the claim that $\left\{\varepsilon_{j} y_{j}\right\}$ is bounded. Assume by contradiction that $\varepsilon_{j}\left|y_{j}\right| \rightarrow \infty$, without loss of generality assume $K\left(\varepsilon_{j} y_{j}\right) \rightarrow K_{\infty}$ and $W\left(\varepsilon_{j} y_{j}\right) \rightarrow W_{\infty}$. Note that $\kappa>K_{\infty}$ and $\omega \geq W_{\infty}$ by $\left(\mathrm{K}_{0}\right)$. Since for any $\varphi \in C_{0}^{\infty}$,

$$
\begin{aligned}
& \Phi_{K_{\infty} W_{\infty}}^{\prime}(w) \varphi \\
& =\left(w^{+}, \varphi^{+}\right)-\left(w^{-}, \varphi^{-}\right)-\int_{\mathbb{R}^{N}} K_{\infty} f(|w|) w \varphi-\int_{\mathbb{R}^{N}} W_{0}|w|^{2^{*}-2} w \varphi \\
& =\lim _{j \rightarrow \infty}\left(\left(w_{j}^{+}, \varphi^{+}\right)-\left(w_{j}^{-}, \varphi^{-}\right)-\int_{\mathbb{R}^{N}}\left(\widehat{K}_{\varepsilon_{j}}(x) f\left(\left|w_{j}\right|\right)-\widehat{W}_{\varepsilon_{j}}(x)\left|w_{j}\right|^{2^{*}-2}\right) w_{j} \varphi\right)=0,
\end{aligned}
$$

consequently, $w=(u, v)$ solves

$$
\begin{cases}-\Delta u+b \cdot \nabla u+u=K_{\infty} f(|w|) v+W_{\infty}|w|^{2^{*}-2} v & \text { in } \mathbb{R}^{N} \\ -\Delta v-b \cdot \nabla v+v=K_{\infty} f(|w|) u+W_{\infty}|w|^{2^{*}-2} u & \text { in } \mathbb{R}^{N}\end{cases}
$$

with the energy

$$
\begin{aligned}
\Phi_{K_{\infty} W_{\infty}}(w) & =\frac{1}{2}\left(\left\|w^{+}\right\|^{2}-\left\|w^{-}\right\|^{2}\right)-\int_{\mathbb{R}^{N}} K_{\infty} F(|w|)-\frac{1}{2^{*}} \int_{\mathbb{R}^{N}} W_{\infty}|w|^{2} \\
& =\int_{\mathbb{R}^{N}} K_{\infty} \widetilde{F}(|w|)+\frac{1}{N} \int_{\mathbb{R}^{N}} W_{\infty}|w|^{2^{*}} \geq c_{K_{\infty} W_{\infty}}
\end{aligned}
$$

here $c_{K_{\infty} W_{\infty}}$ denotes the least energy of (4.16). It follows from $\kappa>K_{\infty}$ and $\omega \geq W_{\infty}$ that $c_{\kappa \omega}<c_{K_{\infty} W_{\infty}}$ by Lemma 3.8. Moreover, by Fatou's lemma,

$\int_{\mathbb{R}^{N}}\left(K_{\infty} \widetilde{F}(|w|)+\frac{1}{N} W_{\infty}|w|^{2^{*}}\right) \leq \lim _{j \rightarrow \infty} \int_{\mathbb{R}^{N}}\left(\widehat{K}_{\varepsilon_{j}}(x) \widetilde{F}\left(\left|w_{j}\right|\right)+\frac{1}{N} \widehat{W}_{\varepsilon_{j}}(x)\left|w_{j}\right|^{2^{*}}\right)$. 
Consequently, by (4.15), (4.16) and Lemma 4.1, we have

$$
c_{\kappa \omega}<c_{K_{\infty} W_{\infty}} \leq \Phi_{K_{\infty} W_{\infty}}(w) \leq \lim _{j \rightarrow \infty} c_{\varepsilon_{j}}=c_{\kappa \omega},
$$

a contradiction.

Thus $\left\{\varepsilon_{j} y_{j}\right\}$ is bounded. Hence, we can assume $x_{j}=\varepsilon_{j} y_{j} \rightarrow x_{0}$. Then $w$ solves

$$
\begin{cases}-\Delta u+b \cdot \nabla u+u=K\left(x_{0}\right) f(|w|) v+W\left(x_{0}\right)|w|^{2^{*}-2} v & \text { in } \mathbb{R}^{N} \\ -\Delta v-b \cdot \nabla v+v=K\left(x_{0}\right) f(|w|) u+W\left(x_{0}\right)|w|^{2^{*}-2} u & \text { in } \mathbb{R}^{N}\end{cases}
$$

with the energy

$$
\begin{aligned}
\Phi_{K\left(x_{0}\right) W\left(x_{0}\right)}(w)= & \frac{1}{2}\left(\left\|w^{+}\right\|^{2}-\left\|w^{-}\right\|^{2}\right) \\
& -\int_{\mathbb{R}^{N}} K\left(x_{0}\right) F(|w|)-\frac{1}{2^{*}} \int_{\mathbb{R}^{N}} W\left(x_{0}\right)|w|^{2^{*}} \\
= & \int_{\mathbb{R}^{N}} K\left(x_{0}\right) \widetilde{F}(|w|)+\frac{1}{N} \int_{\mathbb{R}^{N}} W\left(x_{0}\right)|w|^{2^{*}} \geq c_{K\left(x_{0} W\left(x_{0}\right)\right.},
\end{aligned}
$$

here $c_{K\left(x_{0} W\left(x_{0}\right)\right.}$ denotes the least energy of (4.18). Furthermore, note that $\widehat{K}_{\varepsilon_{j}}(x) \rightarrow K\left(x_{0}\right)$ and $\widehat{W}_{\varepsilon_{j}}(x) \rightarrow W\left(x_{0}\right)$, by Fatou's lemma,

$$
\begin{aligned}
\int_{\mathbb{R}^{N}}\left(K\left(x_{0}\right) \widetilde{F}(|w|)\right. & \left.+\frac{1}{N} W\left(x_{0}\right)|w|^{2^{*}}\right) \\
& \leq \lim _{j \rightarrow \infty} \int_{\mathbb{R}^{N}}\left(\widehat{K}_{\varepsilon_{j}}(x) \widetilde{F}\left(\left|w_{j}\right|\right)+\frac{1}{N} \widehat{W}_{\varepsilon_{j}}(x)\left|w_{j}\right|^{2^{*}}\right) .
\end{aligned}
$$

It follows from (4.15), (4.19) and Lemma 4.1 that

$$
c_{K\left(x_{0}\right) W\left(x_{0}\right)} \leq \Phi_{K\left(x_{0}\right) W\left(x_{0}\right)}(w) \leq \lim _{j \rightarrow \infty} c_{\varepsilon_{j}} \leq c_{K\left(x_{0}\right) W\left(x_{0}\right)} .
$$

This implies that $K\left(x_{0}\right)=\kappa, W\left(x_{0}\right)=\omega$ and $c_{K\left(x_{0}\right) W\left(x_{0}\right)}=c_{\kappa \omega}$, so by Lemma $3.8, x_{0} \in \mathscr{M}$. And it is obvious that one may assume that $y_{j} \in \mathbb{R}^{N}$ is a maximum point of $\left|z_{j}\right|$. Moreover, from the above argument, we readily see that any sequence of such points satisfies $x_{j}=\varepsilon_{j} y_{j}$ converging to some point in $\mathscr{M}$ as $j \rightarrow \infty$.

Next we prove $w_{j} \rightarrow w$ in $H^{2}\left(\mathbb{R}^{N}\right)$. Recall that, by (4.21),

$$
\begin{aligned}
\int_{\mathbb{R}^{N}}\left(K\left(x_{0}\right) \widetilde{F}(|w|)+\frac{1}{N}\right. & \left.W\left(x_{0}\right)|w|^{2^{*}}\right) \\
& =\lim _{j \rightarrow \infty} \int_{\mathbb{R}^{N}}\left(\widehat{K}_{\varepsilon_{j}}(x) \widetilde{F}\left(\left|w_{j}\right|\right)+\frac{1}{N} \widehat{W}_{\varepsilon_{j}}(x)\left|w_{j}\right|^{2^{*}}\right) .
\end{aligned}
$$

Using the Brézis-Lieb lemma, one obtains

$$
\lim _{j \rightarrow \infty} \int_{\mathbb{R}^{N}}\left(\widehat{K}_{\varepsilon_{j}}(x) \widetilde{F}\left(\left|w_{j}-w\right|\right)+\frac{1}{N} \widehat{W}_{\varepsilon_{j}}(x)\left|w_{j}-w\right|^{2^{*}}\right)=0,
$$


which implies that $\left|w_{j}-w\right|_{2^{*}} \rightarrow 0$. Moreover, by the Hölder inequality, one has $\left|w_{j}-w\right|_{p} \rightarrow 0$ in $L^{p}$ for all $p \in\left(2,2^{*}\right)$. Then $\left|w_{j}^{ \pm}-w^{ \pm}\right|_{p} \rightarrow 0$ in $L^{p}$ for all $p \in\left(2,2^{*}\right]$ by the continuity of projection operator. For convenience, denote $\phi_{j}=w_{j}-w$. Remark that $\left\{\phi_{j}\right\}$ is bounded in $E$ and $\phi_{j} \rightarrow 0$ in $L^{p}$ for all $p \in\left(2,2^{*}\right]$. The scale product of (4.13) with $\phi_{j}^{+}$yields

$$
\int_{\mathbb{R}^{N}} A w_{j} \phi_{j}^{+}=\int_{\mathbb{R}^{N}}\left(\widehat{K}_{\varepsilon_{j}} f\left(\left|w_{j}\right|\right) w_{j}+\widehat{W}_{\varepsilon_{j}}\left|w_{j}\right|^{2^{*}-2} w_{j}\right) \phi_{j}^{+}=o(1),
$$

this implies that $\left\langle w_{j}^{+}, \phi_{j}^{+}\right\rangle=o(1)$. Similarly, using the exponential decay of $w$ together with the fact that $w_{j}^{ \pm} \rightarrow 0$ in $L_{\text {loc }}^{q}$ for $q \in\left[2,2^{*}\right)$, it follows from (4.18) that $\left\langle w^{+}, \phi_{j}^{+}\right\rangle=o(1)$. Thus $\left\|\phi_{j}^{+}\right\|=o(1)$, and the same argument shows that $\left\|\phi_{j}^{-}\right\|=o(1)$. We then get $w_{j} \rightarrow w$ in $E$. Similarly to the argument of Lemma 4.3 , we can prove that $w_{j} \rightarrow w$ in $H^{2}\left(\mathbb{R}^{N}\right)$.

Lemma 4.5. Let $w_{j}$ be given as in the proof of Lemma 4.4. Then $\left|w_{j}(x)\right| \rightarrow 0$ as $|x| \rightarrow \infty$ uniformly in $j \in \mathbb{N}$.

Proof. Assume by contradiction that the conclusion of the lemma does not hold. Then by (2.7) there exist $\sigma>0$ and $x_{j} \in \mathbb{R}^{N}$ with $\left|x_{j}\right| \rightarrow \infty$ such that

$$
\begin{aligned}
\sigma & \leq\left|w_{j}\left(x_{j}\right)\right| \leq C_{0}\left(\int_{B_{1}\left(x_{j}\right)}\left|w_{j}\right|^{2}\right)^{1 / 2} \\
& \leq C_{0}\left(\int_{\mathbb{R}^{N}}\left|w_{j}-w\right|^{2}\right)^{1 / 2}+C_{0}\left(\int_{B_{1}\left(x_{j}\right)}|w|^{2}\right)^{1 / 2} \rightarrow 0,
\end{aligned}
$$

since $w_{j} \rightarrow w$ in $H^{2}\left(\mathbb{R}^{N}\right)$, a contradiction.

Lemma 4.6. There are $c, C>0$ and a maximum point $y_{\varepsilon}$ of $\left|z_{\varepsilon}(x)\right|$ such that

$$
\left|z_{\varepsilon}\right| \leq C \exp \left(-\frac{c}{2}\left|x-y_{\varepsilon}\right|\right)
$$

Proof. It follows from $(2.8),\left(\mathrm{F}_{1}\right)$ and Lemma 4.5 that there are $R>0$ and $\delta>0$ such that

$$
\Delta\left|w_{\varepsilon}\right|^{2} \geq \delta\left|w_{\varepsilon}\right|^{2} \quad \text { for all }|x| \geq R \text { and } \varepsilon>0 \text { small. }
$$

Let $\Gamma$ be a fundamental solution to $-\Delta \Gamma+\delta \Gamma=0$. Using the uniform boundedness, we may choose $\Gamma$ so that $\left|w_{\varepsilon}(y)\right|^{2} \leq \Gamma(y)$ holds on $|y|=R$ for all $\varepsilon>0$ small. Set $v_{\varepsilon}=\left|w_{\varepsilon}\right|^{2}-\Gamma$, then

$$
\Delta v_{\varepsilon}=\Delta\left|w_{\varepsilon}\right|^{2}-\Delta \Gamma \geq \delta\left(\left|w_{\varepsilon}\right|^{2}-\Gamma\right)=\delta v_{\varepsilon} .
$$

By the maximum principle, we can conclude that $v_{\varepsilon}(y) \leq 0$ for $|y| \geq R$, i.e. $\left|w_{\varepsilon}(y)\right|^{2} \leq \Gamma(y)$ for $|y| \geq R$. It is well known that there are $c^{\prime}, C^{\prime}>0$ such that

$$
\Gamma(y) \leq C^{\prime} \exp \left(-c^{\prime}|y|\right) \quad \text { for }|y| \geq 1 .
$$


Therefore, there are $c, C>0$ such that $\left|w_{\varepsilon}(x)\right|^{2} \leq C \exp (-c|x|)$ for all $x \in \mathbb{R}^{N}$ and $\varepsilon>0$ small, that is,

$$
\left|z_{\varepsilon}(x)\right| \leq \sqrt{C} \exp \left(-\frac{c}{2}\left|x-y_{\varepsilon}\right|\right)
$$

for all $x \in \mathbb{R}^{N}$ and $\varepsilon>0$ small.

Proof of Theorem 1.1. Going back to $\left(\mathcal{P}_{\varepsilon}\right)$ with the variable substitution $x \mapsto x / \varepsilon, \eta_{\varepsilon}(x):=z_{\varepsilon}(x / \varepsilon)$ is a semiclassical ground state solution of $\left(\mathcal{P}_{\varepsilon}\right)$ for all $\varepsilon>0$ small by Lemma 4.2. Lemma 4.3 shows that conclusion (b) holds. Finally, it is clear that $x_{\varepsilon}:=\varepsilon y_{\varepsilon}$ is a maximum point of $\left|\eta_{\varepsilon}(x)\right|$, and conclusions (c) and (d) follow from Lemmas 4.4 and 4.6, respectively.

\section{REFERENCES}

[1] N. ACKermann, A nonlinear superposition principle and multibump solution of periodic Schrödinger equations, J. Funct. Anal. 234 (2006), 423-443.

[2] A. Ambrosetti, M. Badial and S. Cingolani, Semiclassical states of nonlinear Schrödinger equations, Arch. Ration. Mech. Anal. 140 (1997), 285-300.

[3] A.I. Ávila AND J. YANG, On the existence and shape of least energy solutions for some elliptic systems, J. Differential Equations 191 (2003), 348-376.

[4] _ Multiple solutions of nonlinear elliptic systems, Nonlinear Differential Equations Appl. 12 (2005), 459-479.

[5] T. Bartsch And D.G. De Figueiredo, Infinitely mang solutions of nonlinear elliptic systems, Progr. Nonlinear Differential Equations Appl. Vol. 35, Birkhäuser, Basel, Switzerland (1999), 51-67.

[6] T. Bartsch And Y.H. Ding, Deformation theorems on non-metrizable vector spaces and applications to critical point theory, Math. Nach. 279 (2006), 1267-1288.

[7] J. Byeon and L. Jeanjean, Standing waves for nonlinear Schrödinger equations with a general nonlinearity, Arch. Ration. Mech. Anal. 185 (2007), 185-200.

[8] J. ByeOn AND Z.Q. WANG, Standing waves with a critical frequency for nonlinear Schrödinger equations II, Calc. Var. Partial Differential Equation 18 (2003), 207-219.

[9] D.G. De Figueiredo and J. Yang, Decay, symmetry and existence of solutions of semilinear elliptic systems, Nonlinear Anal. 331 (1998), 211-234.

[10] Y.H. Ding, Variational Methods for Strongly Indefinite Problems, World Scientific Press, 2008.

[11] Y.H. Ding And X.Y. LiU, Semiclassical solutions of Schrödinger equations with magnetic fields and critical nonlinearities, Manuscr. Math. 140 (2013), 51-82.

[12] Y.H. Ding, C. Lee And F.K. Zhao, Semiclassical limits of ground state solutions to Schrödinger systems, Calc. Var. Partial Differential Equations 51 (2014), 725-760.

[13] Y.H. Ding AND B. RuF, Existence and concentration of semiclassical solutions for Dirac equations with critical nonlinearities, SIAM J. Math. Anal. 44 (2012), 3755-3785.

[14] Y.H. Ding And J.C. Wei, Stationary states of nonlinear Dirac equations with general potentials, Rev. Math. Phys. 20 (2008), 1007-1032.

[15] Y.H. Ding, J.C. Wei And T. Xu, Existence and concentration of semi-classical solutions for a nonlinear Maxwell-Dirac system, J. Math. Phys. 54 (2013), 061505.

[16] M. Esteban and E. SÉRÉ, Stationary states of the nonlinear Dirac equation: A variational approach, Comm. Math. Phys. 171 (1995), 250-323. 
[17] A. Floer And A. Weinstein, Nonspreading wave packets for the cubic Schrödinger equation with a bounded potential, J. Funct. Anal. 69 (1986), 397-408.

[18] C.F. GUI, Existence of multi-bump solutions for nonlinear Schrödinger equations via variational method, Commun. Partial Differential Equations 21 (1996), 787-820.

[19] D. Gilbarg and N.S. Trudinger, Elliptic Partial Differential Equations of Second Order, Springer, Berlin (1983).

[20] S.Y. He, R.M. Zhang AND F.K. ZhaO, A note on a superlinear and periodic elliptic system in the whole space, Comm. Pure. Appl. Anal. 10 (2011), 1149-1163.

[21] L. JEANJEAN AND K. TANAKA, Singularly perturbed elliptic problems with superlinear or asymptotically linear nonlinearities, Calc. Var. Part. Diffe. Equ. 21 (2004) 287-318.

[22] W. KRyszewki And A. Szulkin, Generalized linking theorem with an application to semilinear Schrödinger equation, Adv. Differential Equations 3 (1998), 441-472.

[23] Y.Y. LI, On singularly perturbed elliptic equation, Adv. Differential Equations 2 (1997), 955-980.

[24] G. Li And J. YAng, Asymptotically linear elliptic systems, Commun. Partial Differential Equations 29 (2004), 925-954.

[25] P.L. Lions, The concentration compactness principle in the calculus of variations. The locally compact case, Part II, Ann. Inst. H. Poincaré Anal. Non Linéaire 1 (1984), 223-283.

[26] F. LiaO, X.H. TANG AND J. Zhang, Existence of solutions for periodic elliptic system with general superlinear nonlinearity, Z. Angew. Math. Phys. 66 (2015), 689-701.

[27] Y.G. Он, On positive multi-lump bound states of nonlinear Schrödinger equations under multiple well potential, Commun. Math. Phys. 131 (1990), 223-253.

[28] M. DEL PINo AND P. FELmer, Semi-classical states of nonlinear Schrödinger equations: a variational reduction method, Math. Ann. 324 (2002), 1-32.

[29] B. Sirakov, On the existence of solutions of Hamiltonian elliptic systems in $\mathbb{R}^{N}$, Adv. Differential Equations 5 (2000), 1445-1464.

[30] A. Szulkin And T. Weth, Ground state solutions for some indefinite problems, J. Funct. Anal. 257 (2009), 3802-3822.

[31] X.F. WANG, On concentration of positive bound states of nonlinear Schrödinger equations, Comm. Math. Phys. 153 (1993), 229-244.

[32] J. WANG, J.X. XU AND F.B. ZHANG, Existence of semiclassical ground-state solutions for semilinear elliptic systems, Proc. Roy. Soc. Edinburgh Sect. A 142 (2012), 867-895.

[33] L.R. XIA, J. ZHANG AND F.K. ZhaO, Ground state solutions for superlinear elliptic systems on $\mathbb{R}^{N}$, J. Math. Anal. Appl. 401 (2013), 518-525.

[34] M.B. YAng, W.X. Chen AND Y.H. Ding, Solutions of a class of Hamiltonian elliptic systems in $\mathbb{R}^{N}$, J. Math. Anal. Appl. 352 (2010), 338-349.

[35] F.K. ZhaO, L.G. ZhaO AND Y.H. Ding, Infinitly mang solutions for asymptotically linear periodic Hamiltonian system, ESAIM: Control, Optim. Calc. Var. 16 (2010), 77-91.

[36] _ Multiple solutions for asympototically linear elliptic systems, NoDEA Nonlinear Differential Equations Appl. 15 (2008), 673-688.

[37] _ Multiple solution for a superlinear and periodic ellipic system on $\mathbb{R}^{N}, \mathrm{Z}$. Angew. Math. Phys. 62 (2011), 495-511.

[38] _ A note on superlinear Hamiltonian elliptic systems, J. Math. Phys. 50 (2009), 112702.

[39] F.K. ZhaO AND Y.H. Ding, On Hamiltonian elliptic systems with periodic or non-periodic potentials, J. Differential Equations 249 (2010), 2964-2985.

[40] R.M. ZhANG, J. Chen AND F.K. ZhaO, Multiple solutions for superlinear elliptic systems of Hamiltonian type, Discrete Contin. Dyn. Syst. Ser. A 30 (2011), 1249-1262. 
[41] J. ZhANG, W.P. QIN AND F.K. ZhaO, Existence and multiplicity of solutions for asymptotically linear nonperiodic Hamiltonian elliptic system, J. Math. Anal. Appl. 399 (2013), 433-441.

[42] J. Zhang, X.H. Tang And W. Zhang, Ground-state solutions for superquadratic Hamiltonian elliptic systems with gradient terms, Nonlinear Anal. 95 (2014), 1-10.

[43] _ Semiclassical solutions for a class of Schrödinger system with magnetic potentials, J. Math. Anal. Appl. 414 (2014), 357-371.

[44] _ On semiclassical ground state solutions for Hamiltonian elliptic systems, Appl. Anal. 94 (2015), 1380-1396.

[45] J. Zhang, W. Zhang and X.L. XIE, Existence and concentration of semiclassical solutions for Hamiltonian elliptic system, Comm. Pure Appl. Anal. 15 (2016), 599-622.

[46] M. Willem, Minimax Theorems, Birkhäuser, Berlin, 1996.

Manuscript received September 11, 2015

accepted January 9, 2016

JIAN ZHANG

School of Mathematics and Statistics

Hunan Univeristy of Commerce

Changsha, 410205 Hunan, P.R. CHINA

E-mail address: zhangjian433130@163.com

Xianhua Tang and Wen Zhang

School of Mathematics and Statistics

Central South University

Changsha, 410083 Hunan, P.R. CHINA

E-mail address: tangxh@mail.csu.edu.cn, zwmath2011@163.com 